\title{
Micromechanical Modelling of Stress Waves in Rock and Rock Fractures
}

\author{
R. Resende $\cdot$ L. N. Lamas $\cdot$ J. V. Lemos • \\ R. Calçada
}

Received: 6 January 2010/Accepted: 7 April 2010/Published online: 1 May 2010

(c) Springer-Verlag 2010

\begin{abstract}
The goal of this paper is to simulate the interaction of stress waves and rock fractures in a particle micromechanical model. Stress waves travelling in fractured rock masses are slowed down and attenuated by natural heterogeneities, voids, microcracks and, above all, by faults and fractures. Considerable laboratory and theoretical investigation have uncovered the major aspects of this phenomenon, but models that cover the core mechanisms of the wave propagation in rock masses are necessary to investigate aspects of wave-fracture interaction, which are not completely clear, and in the future simulate full-scale real problems. The micromechanical model is based on the particle discrete element model that reproduces rock through a densely packed non-structured assembly of 2D disks with point contacts. The model of a hard rock core is developed and an irregular rock joint is generated at midheight. A new contact constitutive model is applied to the particles in the joint walls. Numerical static joint compression tests are performed and a typical hyperbolic stress-displacement curve is obtained. Conditions for good quality wave transmission through non-jointed unorganized particulate media are determined, hybrid static-dynamic boundary conditions are established and plane waves are emitted into the compressed joint. The transmitted and
\end{abstract}

R. Resende $(\bowtie) \cdot$ L. N. Lamas · J. V. Lemos

National Laboratory for Civil Engineering (LNEC),

Av. Brasil, 101, 1700-066 Lisbon, Portugal

e-mail: rresende@lnec.pt

URL: http://www.lnec.pt

R. Calçada

Faculty of Engineering of the University of Oporto (FEUP),

R. Doutor Roberto Frias s/n, 4200-465 Porto, Portugal

e-mail: ruiabc@fe.up.pt

URL: http://www.fe.up.pt reflected waves are extracted and analysed. Joint dynamic stiffness calculated according to the hypotheses of the Displacement Discontinuity Theory shows to increase with the static joint compression until the joint is completely closed. Still in its early stages of application, this rock micromechanical model enables the joint behaviour under static and dynamic loading to be analysed in detail. Its advantages are the reproduction of the real mechanics of contact creation, evolution and destruction and the possibility of visualizing in detail the joint geometry changes, which is hard to accomplish in the laboratory.

Keywords Rock joint - Rock dynamics - Stress wave · Discrete element method $\cdot$ Micromechanical model

\section{Introduction}

Rock joints are the main obstacle to stress wave propagation in rock masses. Through several kinds of interaction, waves' peaks are diminished, propagation is delayed and frequencies are selectively filtered. The interaction is twoway because waves with sufficient amplitude can too change the state of the joints. Voids, micro-fractures and schistosity on the rock matrix are also responsible for attenuation of stress waves but in hard rock, their role is usually secondary.

Schoenberg (1980) managed to integrate the wave equation of $\mathrm{P}$ and $\mathrm{S}$ waves with arbitrary angles of incidence, expressing the joint dynamic response as a function of joint compliance. Later, Pyrak-Nolte et al. (1990) expanded Schoenberg's solution to the case of discontinuities filled with viscous fluids (Kelvin and Maxwell models). In Pyrak-Nolte's formulation, joint response is expressed by means of the dynamic stiffness, the inverse of 
joint compliance. In the Displacement Discontinuity Theory (DDT) stress (and therefore velocity) is continuous across the joint, while displacement is proportional to stress since normal and shear stiffness are finite and constant. DDT provides solutions for 3D shear and normal wave interaction with rock joints and has shown to be accurate when compared with results for normally incident shear and normal waves.

For each type of body wave interacting with a joint as illustrated in Fig. 1, DDT provides the amplitude and phase delay of each of the reflected and transmitted waves. For the general case, a $4 \times 4$ system of equations must be solved, but for normally incident waves $\left(\theta_{1}=0\right.$ or $\left.\phi_{1}=0\right)$ no wave conversion occurs and the amplitude of the reflected $R(\omega)$ and transmitted $T(\omega)$ waves on the frequency domain are obtained from the incident wave $I(\omega)$, by:

$$
\begin{aligned}
& |R(\omega)|=|I(\omega)|\left[\frac{4\left(\frac{\omega Z_{x}}{K_{x}}\right)^{2}}{4\left(\frac{\omega Z_{x}}{K_{x}^{\mathrm{dyn}}}\right)^{2}+1}\right]^{1 / 2}, \\
& |T(\omega)|=|I(\omega)|\left[\frac{1}{4\left(\frac{\omega Z_{x}}{K_{x}^{\mathrm{dyn}}}\right)^{2}+1}\right]^{1 / 2}
\end{aligned}
$$

where $\omega$ is angular frequency and the subscript $x$ represents either compressive or shear wave impedance of the rock or joint stiffness: $K_{x}$ may be $K_{n}^{\mathrm{dyn}}$ or $K_{s}^{\mathrm{dyn}}$ and $Z_{x}$ may be $Z_{p}$ or $Z_{s}$. The parameter that controls the amount of transmission and reflection of the compressive wave is the relation between the wave angular frequency and the joint dynamic or seismic specific stiffness $K_{n}^{\text {specific }}$ expressed by:

$K_{n}^{\text {specific }}=\frac{K_{n}^{\text {dyn }}}{\omega Z_{p}}$.

A change in the wave amplitude causes a phase shift that corresponds to a time delay $t_{g}$ : $t_{g}=\frac{\frac{2 K_{x}^{\mathrm{dyn}}}{Z_{x}}}{\left(\frac{2 K_{x}^{\mathrm{dyn}}}{Z_{x}}\right)^{2}+\omega^{2}}$.

In laboratory tests of joint-wave interaction, the frequency content of the incident and transmitted waves and the impedance of the rock material can be measured directly. Therefore, the only unknown variable in Eqs. 1, the dynamic stiffness $K_{n}^{\text {dyn }}$, can be determined. Finally, and given that the system is elastic, there is no loss of energy associated to the wave reflection and transmission: $|R|^{2}+|T|^{2}=|I|^{2}$.

Figure 2 displays the plots of Eqs. 1a and b. When frequency is low in comparison to the specific stiffness the wave is completely transmitted, as this corresponds to a wave with null frequency. On the other hand, when the frequency is high the wave is completely reflected.

Nolte et al. (2000) determined that for the DDT to be valid fracture extension must be much larger and fracture aperture much smaller than wavelength; asperity spacing must be much smaller than wavelength, and the distribution of contacts must be approximately uniform.

Dynamically speaking the first and second conditions approximately define what a fracture is. If fracture length is smaller or in the same order of the wavelength, the wave will "sense" it as a small crack. In addition, if the crack opening is not much smaller than the wavelength, the wave will loose all its features when crossing it. The two last conditions must be met to avoid resonance scattering at the voids between the contacts of the walls.

Micromechanical models aim at predicting the mechanical response of rock by simulating the microscopic mechanisms of deformation and failure. The major advantage is that the material is modelled without resorting to constitutive models that are getting increasingly complex. If the fundamental hypotheses that drive the microscopic behaviour of the media are correctly and efficiently (a)

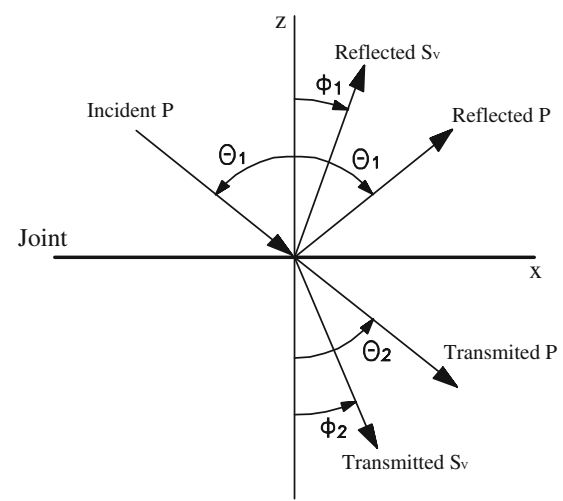

(b)

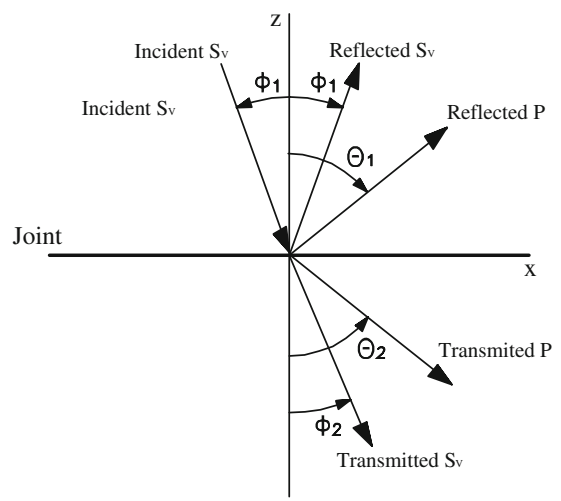

(c)

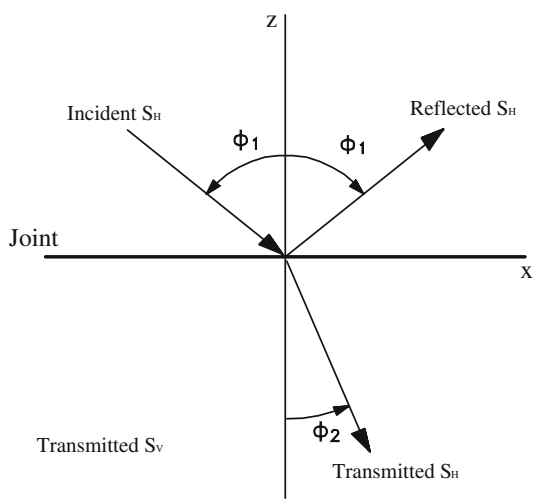

Fig. 1 Transmitted and reflected waves at discontinuities: compression wave (a), vertical shear wave (b) and horizontal shear wave (c) 


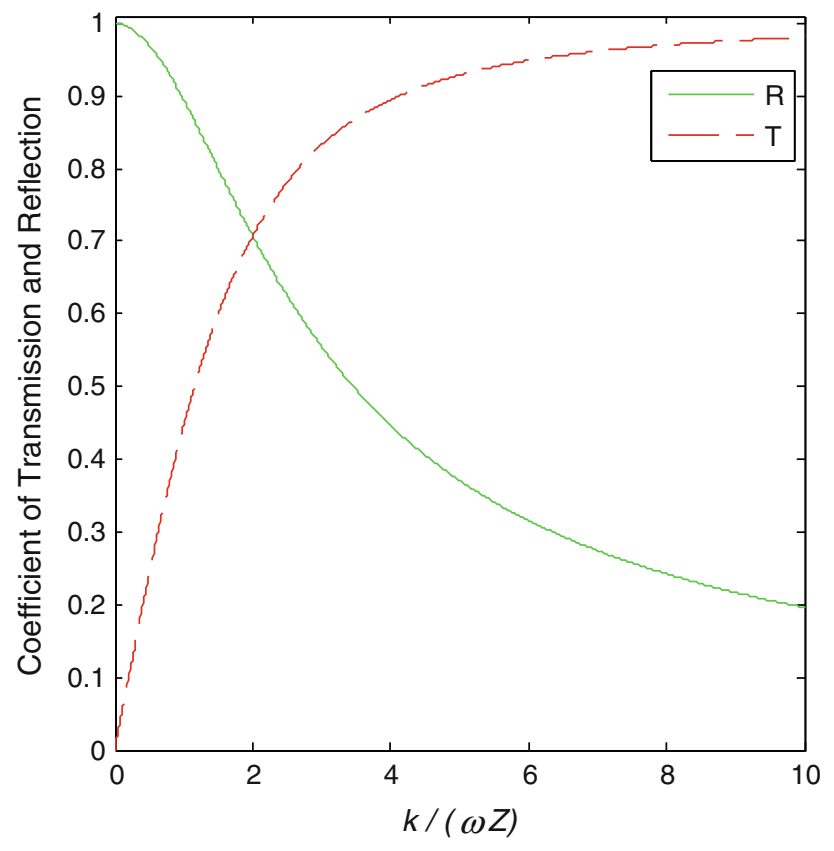

Fig. 2 Magnitude of transmission and reflection ratios as functions of nondimensional frequency for $\mathrm{P}$ and $\mathrm{S}$ waves normally incident to an elastic discontinuity

implemented, the interaction of a few simple mechanisms covers the macroscopic response of the material under a broad range of situations. One of the first microscopic level models is the lattice method. Schlangen and van Mier (1994), among other authors, tested different arrangements of lattice structures to model the response of sandstone.

In the Bonded Particle Model (BPM), based on the Discrete Element Method, DEM (Cundall and Strack 1979), response of rock under a number of situations can be simulated by assemblies of a large number of circular or spherical particles with finite radius bonded by ball-ball contacts. The relation between the models properties (assembly arrangement, particles' radius and contact characteristics) on a microscopic level and rock macroscopic properties such as compressive strength or Young's modulus is not direct, as there is no complete theory to relate micro and macroscopic properties. The constant and steady growth of computational capacities and a number of very successful applications of BPM to model complex rock behaviour has promoted the utilization of this method (e.g. Potyondy et al. 1996; Potyondy and Cundall 2004).

Not much attention, however, has been dedicated to the modelling of rock dynamic behaviour using particle models. In the field of geophysics, some authors have used particle codes to model wave propagation in large scale, from hundreds of meters to tens of kilometres (Toomey and Bean 2000; Abe et al. 2004). However, the model in question employed regular assemblies of particles, which do not mimic with realism rock more complex behaviour.
Matsuoka et al. (2003) also used a regular arrangement of disks to model a Hopkinson bar test of rock capturing some of the main features of the experiment, namely the detachment of a piece of the bar extremity ejected due to the reflected tension wave. Hentz et al. (2004) employed a $3 \mathrm{D}$ particle model to investigate the effect of strain rate in the compressive and tensile strength of rock and several authors have used particle models to model blasting in several aspects. Kim et al. (1997) and Donzé et al. (1997) modelled the crack generation caused by a blast charge in a cylindrical borehole, Donzé and Bernasconi (2004) modelled a shaft sinking blast in three dimensions obtaining patterns of rock damage around the shaft. Kim et al. (2006) investigated the influence of joint direction and spacing at a tunnel contour blasting. These works employ particle methods on blasting and rock dynamic applications managing to qualitatively capture the major phenomena at stake, namely fracture creation and propagation, rock fragmentation, movement of the blasted rock and damage due to high dynamic stress. However, the propagation of elastic stress waves outside the zone of rock fragmentation is not a relevant issue in these cases and has not been addressed.

Hazzard and Young (2004) used $\mathrm{PFC}^{3 \mathrm{D}}$ to reproduce the anisotropic damage inflicted to a sandstone sample by triaxial deviatoric loading. This is the only work, to the knowledge of the authors, which investigates some of the characteristics of the propagation of stress waves in unorganized particle models of rock.

Interaction of the stress waves with rock fractures have been explicitly modelled in block DEM codes. Works of Lemos (1987) and later Cai (2001) apply the 2D DEM program UDEC to plane waves normally incident on fractures. The fractures have linear elastic stiffness and the model is able to reproduce the frequency dependence of the transmission and reflection ratio. The fractures are represented as contacts between blocks, and their mechanical attributes are shear and normal stiffness. This characterization of fractures is, however, far from reality. Fractures are irregular present normal and shear non-linear response and show different stiffness values according to the intensity and velocity of the loading. More sophisticated block-to-block contact models have been developed, including some of these effects (Zhao and Cai 2001), but the response is represented by a constitutive model that does not include explicitly the real cause of the fracture's behaviour namely asperity distribution, deformation and degradation with normal and shear load.

There have been a few applications of particle models static shear behaviour of rock joints that represent joint roughness explicitly, but work on the characterization of rock joints under normal loading has been found in the literature. 
This paper is dedicated to the application of BPM to the modelling of wave propagation in rock masses. The purpose is to investigate the capabilities of this numerical method on modelling wave propagation in rock masses and rock fractures at the microscopic level. The model mimics the deformation of asperities and allows the observation in close detail of the passage of the waves across the fractures. The focus is on the simplest case, i.e. propagation of low amplitude high frequency ( $\mathrm{kHz}$ range) plane compressive waves normally incident on fractures previously loaded by static compressive stress. Static loading forces the joint to close leading to the increase of the contact area. Therefore, transmissivity of the joint also grows.

A particle model of the joint that reproduces a generic rock joint under normal stress and emulates the increase in contact area and corresponding growing stiffness with applied stress is developed. Then, transmitted and reflected waves that result from the interaction of waves and the compressed joint are analysed.

\section{Simulation of Rock and Rock Fractures with BPM}

\subsection{Generation of a BPM for Hard Rock}

The procedure employed to generate the particle assembly is adapted from Potyondy and Cundall (2004). To model wave propagation and capture the complete waveforms, a long $(90 \times 600 \mathrm{~mm})$ rock specimen is produced. It is composed by 34,773 particles with radii that vary uniformly between 0.50 and $0.83 \mathrm{~mm}$ (average diameter is $1.33 \mathrm{~mm}$ ). Disk density is set to $3,150 \mathrm{~kg} / \mathrm{m}^{3}$. Given that the porosity of the packing is $14.3 \%$, continuum-equivalent porosity is $2,700 \mathrm{~kg} / \mathrm{m}^{3}$ (this value is relevant only in the dynamic part of the study, as self-weight is not considered in the static calculation). As to the ball-ball contact, two kinds are possible: simple contact bonds and parallel bonds, the latter being able to offer resistance to rolling between particles. Since there is very little experience with wave propagation in granular assemblies, it is decided to employ contact bonds, which having simpler individual response results in a more predictable macroscopic model. Contacts normal stiffness is set to $6.20 \times 10^{10} \mathrm{~N} / \mathrm{m}, 2.5$ times the shear stiffness.

\subsection{Boundary Conditions}

To define the model borders where loads and other boundary conditions are applied, a strip of balls is identified at the top, sides and bottom of the sample, as shown in Fig. 3. The width of the strip is defined with enough size to leave no part of the model unbounded.

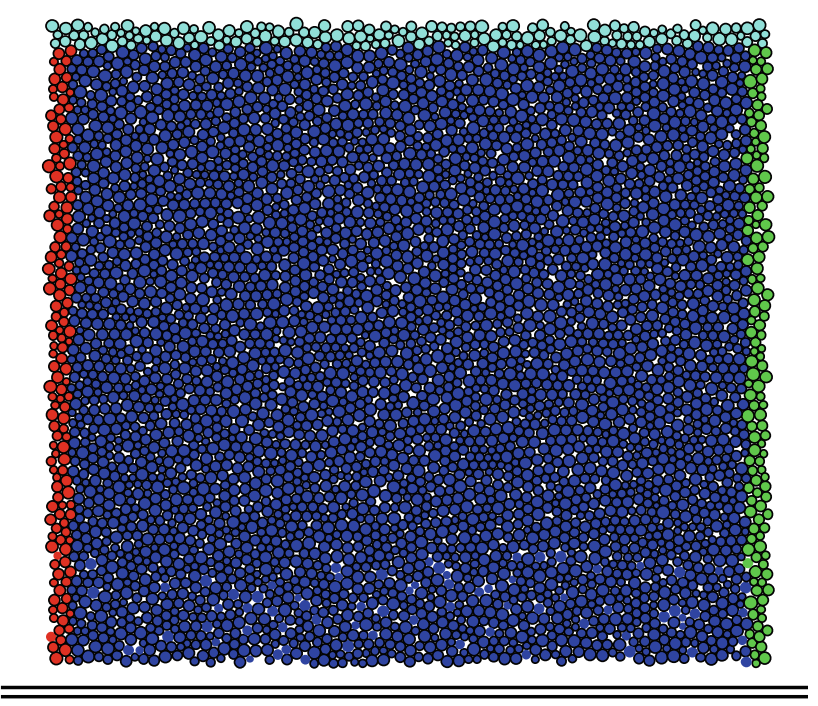

Fig. 3 Top and lateral boundaries of the model

Since stress is a concept valid only in continuous media it must be translated into forces that are applied to the balls centres. If a stress value $\sigma$ is to be applied at a cylindrical particle with unitary height, the equivalent force $\left(F_{\text {ball }}\right)$ must take into account the transversal area of the particle:

$F_{\text {ball }}=\sigma \cdot A_{\text {ball }} \Leftrightarrow F_{\text {ball }}=\sigma \cdot 2 \cdot r_{\text {ball }}$.

The passage from stress at the boundary to forces applied to the balls must also take into account the border's depth (measured in terms of balls). In regular rectangular packings, the boundary may be easily set to be one ball deep. Thus, no correction is necessary. In irregular packings with thicker boundaries, the boundary depth correction factor $b f_{\text {depth }}$ is:

$b f_{\text {depth }}=\frac{l_{\text {bound }}}{2 \cdot r_{\text {mean }}} \cdot \frac{1}{n b_{\text {bound }}}$

where $l_{\text {bound }}$ is the boundary length and $n b_{\text {bound }}$ is the number of balls in that boundary. The force in each particle is given by the product of Eqs. 4 and 5:

$F_{\text {ball }}=b f_{\text {depth }} \cdot \sigma \cdot A_{\text {ball }} \Leftrightarrow F_{\text {ball }}=\frac{l_{\text {bound }}}{r_{\text {mean }}} \cdot \frac{1}{n b_{\text {bound }}} \cdot \sigma \cdot r_{\text {ball }}$.

The present model has a $90 \mathrm{~mm}$ width the average ball radius being $0.665 \mathrm{~mm}$. One "average" row of balls has thus $90 /(0.665 \times 2) \approx 69$ balls. The top boundary has 164 balls, so the force in each particle becomes

$F_{\text {ball }}=b f_{\text {depth }} \cdot \sigma \cdot A_{\text {ball }} \Leftrightarrow F_{\text {ball }}=\frac{69}{164} \cdot \sigma \cdot 2 \cdot r_{\text {ball }}$.

\subsection{Model Static Properties}

An unconfined compression test of the assembly shows Young's modulus to be fairly constant in the range of 
compressive stresses of interest (0-120 MPa) with an average value of $43.5 \mathrm{GPa}$ and a standard deviation of $0.54 \mathrm{MPa}$, while Poisson's ratio is approximately 0.2 (Fig. 4). In this test balls at the lateral boundary can rotate and move in all directions. A null-velocity condition is applied to the bottom boundary balls. Balls at the top boundary are not allowed to rotate and move in the horizontal direction. A vertical downwards force is applied to enforce the normal stress in the model.

The slight increase in the Young's modulus with normal stress is consequence of creation of new contacts. Since the goal of this simulation is in a first stage to isolate the influence of the BPM fabric in the wave progression, and after to focus on the phenomena that occur at the joint contacts are not allowed to fail. This is achieved by setting their bond strength to very high values. This decision is not free from criticism. In a joint compression test, it is the failure at asperity level that after a certain amount of compression causes non-linearity, but this is dealt with in another way, as will be shown ahead. In addition, microfracturating or plastic damage can occur in the rock material that supports the asperities which means that it is difficult to define exactly where the material in the rock core ends and the joint begins.

\subsection{Simulation of Joint Behaviour}

Rock joints' behaviour under normal loading has been the object of less attention than shear behaviour. The main reason is that rock masses under low to medium stress fail mainly due to joint tangential movement. It is in seismic and hydraulic scenarios that joint deformation under normal loads is particularly important because joint aperture commands hydraulic conductivity and wave transmissivity. The need for models that realistically incorporate the normal behaviour of joints increases as dynamic and hydro-mechanical coupled analysis become more frequent driven by the modelling of hydrocarbon reserves, thermal energy production and storage of $\mathrm{CO}_{2}$ and radioactive waste.

The stress-displacement curve of a compression test displays the distinct hyperbolic shape, as shown in Fig. 5. The curve shows initial low stiffness stepping up as the joint closure increases. In the beginning of the test, when the top part of the specimen is placed over the lower part, the area of contact between both halves may be very small. The load applied in each half of the specimen is transmitted through these few contact points, which are under large local stresses. They deform significantly and/or are partially or totally crushed, depending of their strength to stiffness ratio. The increase in displacement that corresponds to each load increment is large in this initial part of the curve, leading to an almost flat curve.

As joint compression goes up, walls distance shortens, more asperities from both sides come in contact, the load gets distributed over a larger area and the stress in each contact zone diminishes. The deformation of an asperity also increases its own area but this effect contributes much less than new contacts to the global increase in contact area and therefore to stiffness. As the load is supported by more
Fig. 4 Vertical stress versus vertical strain (a) and Young's modulus (b) (a)

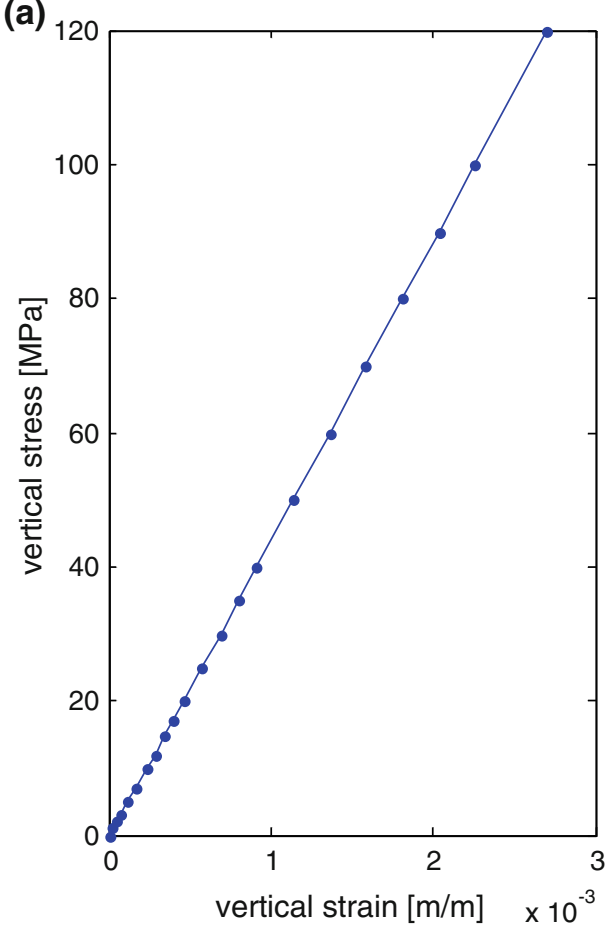

(b)

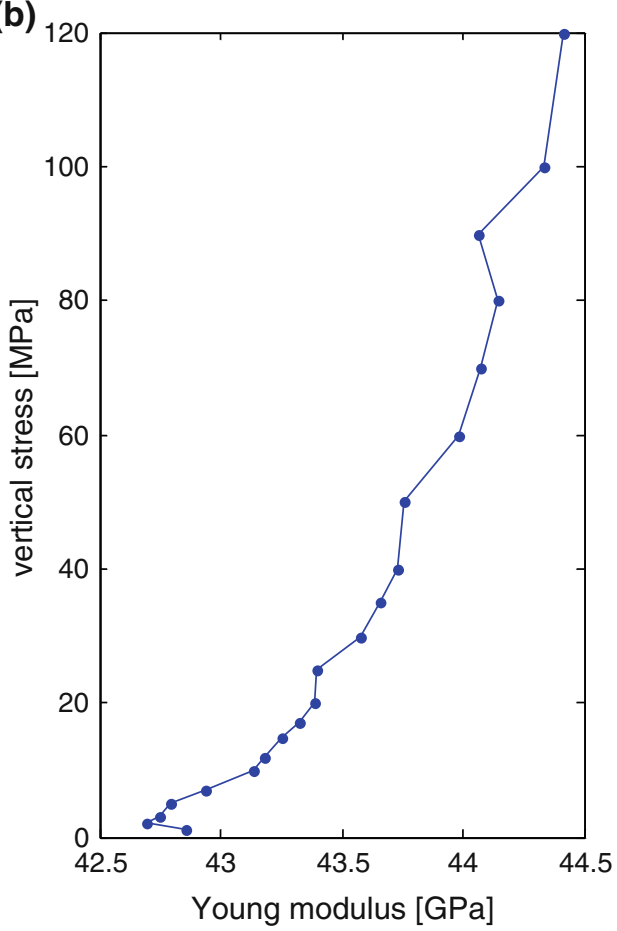




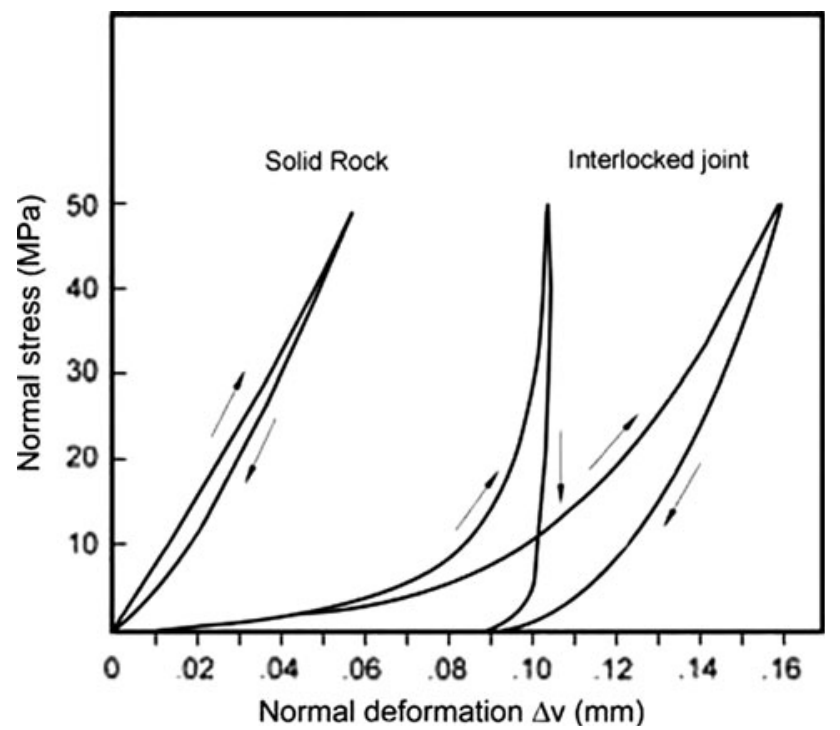

Fig. 5 Non-linear, hysteretic normal-closure behaviour of rock joint (Bandis et al. 1983)

and more contacts, the resistance to closure grows and the stress-displacement curve gets steeper. The rock material that supports the asperities also deforms, which makes it harder to distinguish where the effect of the rock joint deformation ends and that of the rock material starts.

As mentioned in the introduction, particle models have been used to model static tests of rough undulating rock joints in shear (Kabeya 1999; Cundall 2000; Kusumi et al. 2005) but not in compression. Gutierrez and Barton (1994) employed UDEC to represent a shear test of a rock joint. The joint topography is described by a continuous, irregular line that mimics typical JRC profiles (Bandis et al. 1983). The model allowed the investigation of the micromechanics of the shear failure of these joints and also the evolution of hydraulic aperture. Recently, Karami and Stead (2008) used the hybrid FEM/DEM code ELFEN to model the shear behaviour of several JRC profiles under varying normal stresses. Several other authors (Toomey 2001; Park et al. 2004; Min et al. 2005) have employed block discrete element and particle models in a larger scale to represent large volumes of rock masses that include several joint sets. The topography of discontinuities' walls is not represented as joints are defined by assigning lower contact strengths and/ or stiffness to particles that lay upon or on the vicinity of the line (in 2D) or plane (in 3D) that define the joint. Nevertheless, macroscopic influence of joint sets in rock mass properties is evident in these models.

\subsection{Normal Behaviour of the Jointed Synthetic Rock Specimen}

Micromechanical models can be used to emulate the geometry of joints to a degree of detail that is limited by the means to characterize their geometry and the hardware capacity. If particles with a mean radius of $0.25 \mathrm{~mm}$ are employed (granite typical grain size is greater than $0.5 \mathrm{~mm})$, a $100 \times 100 \mathrm{~mm} 2 \mathrm{D}$ sample can be modelled by approximately 50,000 particles. It has been found that a model with this level of detail is appropriate to represent the basic features of a joint under static and dynamic loadings being manageable by a standard personal computer.

To simulate a real test, both the stress-displacement curve and a detailed surface characterization of both joint surfaces that can be reproduced by the particle assembly are necessary. As these are not available, a generic rock joint geometry is generated and tested. Another feature of this simulation is that, given that $3 \mathrm{D}$ runs are many times more time-consuming than $2 \mathrm{D}$ runs, the simulation is run in 2D.

Previous utilizations of BPM to model rock joint shear tests used various strategies to generate fractures. Cundall's (2000) approach to generate the surfaces for the shear test of a rough joint was to use a sum of varying wavelength sine waves, thus replicating the several orders of roughness. Kusumi et al. (2005) reproduced different JRC profiles by erasing particles from a rock model. Another method is to create a realistic fracture surface by submitting a notched rock core to a tensile load (possibly in combination with shear) until complete separation of the two halves. The employed method, which is also the most straightforward one, is to simply delete enough balls in the middle of the synthetic specimen until there is no contact between the two halves. The resulting joint surfaces are potentially less rich, but have the advantage of being very predictable, planar and with small irregularities (Fig. 6).

Inter-particle contact drives the macroscopic behaviour of synthetic rock samples. Microproperties are not related to measurable properties like Young's modulus or Poisson's ratio through analytical laws, but by empirical relations that have been established through experience.

When it comes to the simulation of the microscopic properties of joint wall-to-wall contacts, no previous experience is available. The displacement in rock joints under compression is mostly caused by elastic deformation of the asperities followed by their partial or total crushing together with deformation of the rock that supports the asperities. It is then postulated that after suffering a certain amount of deformation and crushing the contact area of each asperity increases and consequently the same happens to the contact stiffness.

In the current representation of the rock surface each peak is represented by a small number of particles (see Fig. 6b), thus keeping the number of balls in the model manageable through the whole static and dynamic calculations. Peak deformation, crushing and hardening must be 
Fig. 6 Generation of joint surface. Balls marked for deletion (a); detail of the surface after deletion of marked balls (b) (a)

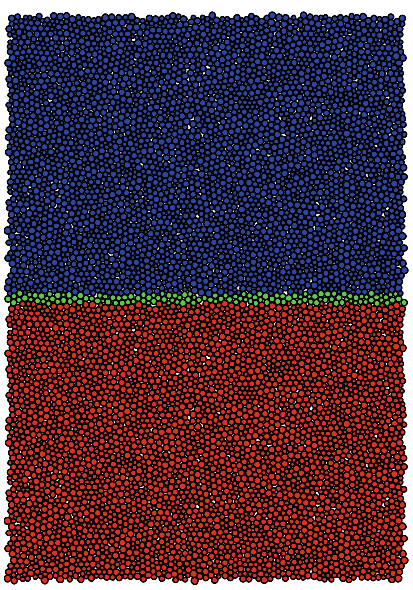

(b)

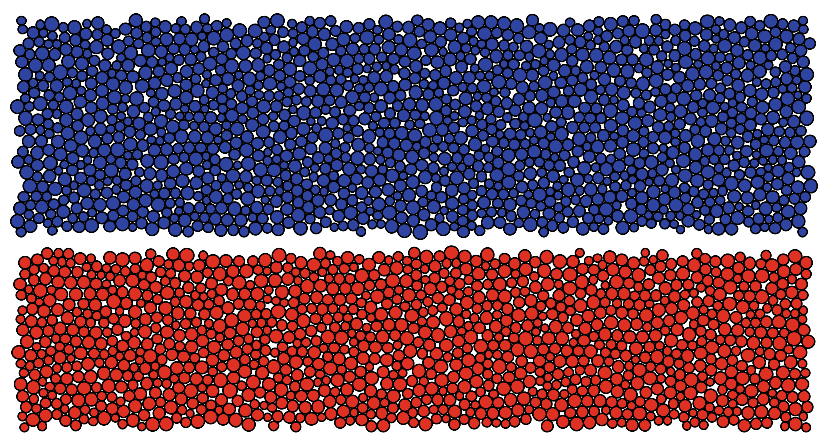

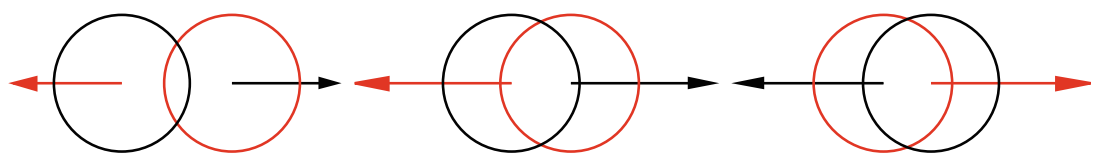

Fig. 7 Green arrow denotes repulsion exerted by green ball on red ball and vice versa. As balls' centres pass through each other, direction of repulsion is inverted

simulated at each ball. A new contact constitutive model is developed and compiled, resulting in a $\mathrm{C}^{++}$dynamic linked library that is invoked by PFC.

It was found that if the contact properties of the rock material are used at the contact between balls in each joint wall, the first balls to come into compression withstand very high values of contact force and do not deform enough to allow new contacts to be created. On the other hand, if low contact stiffness is employed inter-penetration of balls in opposing faces is such that their centres cross each other and the direction of repulsive forces reverses, pushing the sample halves against each other, as represented in Fig. 7.

The constitutive model developed in order to overcome this problem is bi-linear in shear and compression, with no tensile strength and a Mohr-Coulomb strength criterion in shear. Its force versus ball overlap curve is shown in Fig. 8.

The contact initial and final stiffness are set as a percentage of the stiffness of the contacts in the rock material $\left(k_{n}\right)$ via the parameters $d_{k i}$ and $d_{k f}$. The point where stiffness value increases, simulating a fast increase of area in the contact, is defined as a percentage of the radius of the smaller ball of the pair.

To maintain the model calibration as simple as possible, shear and compressive stiffness evolve in parallel, and the final stiffness is equal to the rock matrix stiffness, which corresponds to $d_{k f}=1$. The behaviour is then defined by two parameters: initial percentage of the rock stiffness $d_{k i}$ and percentage of the radius of the smaller ball in contact that triggers hardening $d_{\text {radius }}$.

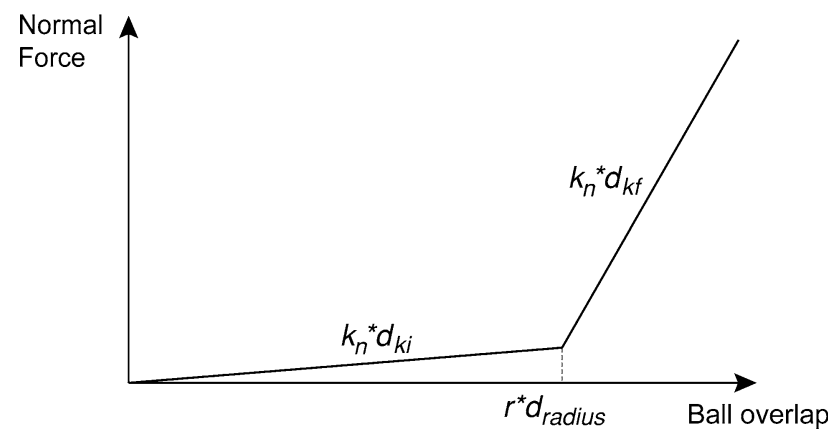

Fig. 8 Joint constitutive model diagram

Strategies to represent asperity degradation, such as ball radius decrease, ball deletion or ball breakage have shown to be either inadequate or more difficult to implement adequately.

The normal stress versus normal displacement curve for the parameters $d_{k i}=1 \%, d_{\text {radius }}=10 \%$ and $d_{k f}=100 \%$ is depicted in Fig. 9a. The stress-displacement curve shows the main characteristics of rock joint normal tests. These are initial low stiffness due to a small number of contacts followed by a gradual increase of the contact area that leads to an almost vertical graph. This reflects the creation of new contacts and the increase of stiffness in existing ones. In Fig. 9b, the stiffness evolution is represented. Its evolution is also according to the experimental results. The abrupt variations, also observed in real tests, are due to the high sensibility of the stiffness to very small features on the 
Fig. 9 Normal stress versus joint closure (a) and tangent joint stiffness (b) (a)

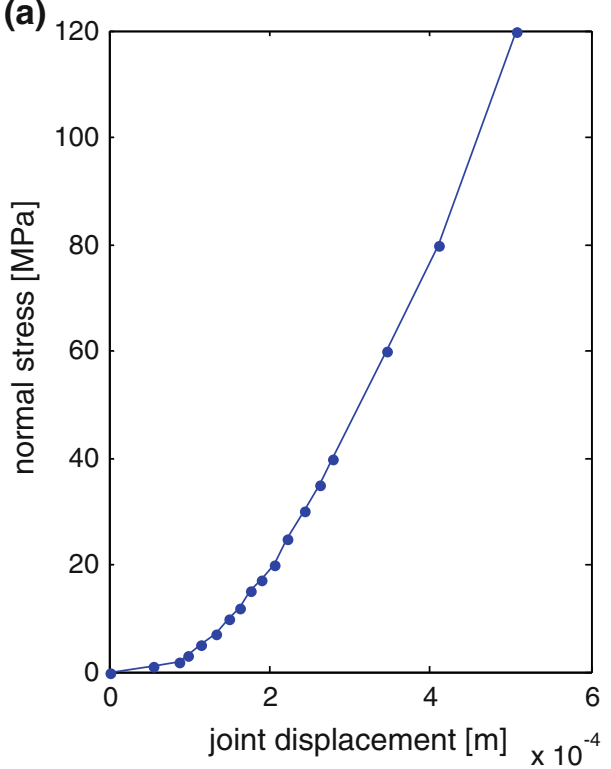

(b)

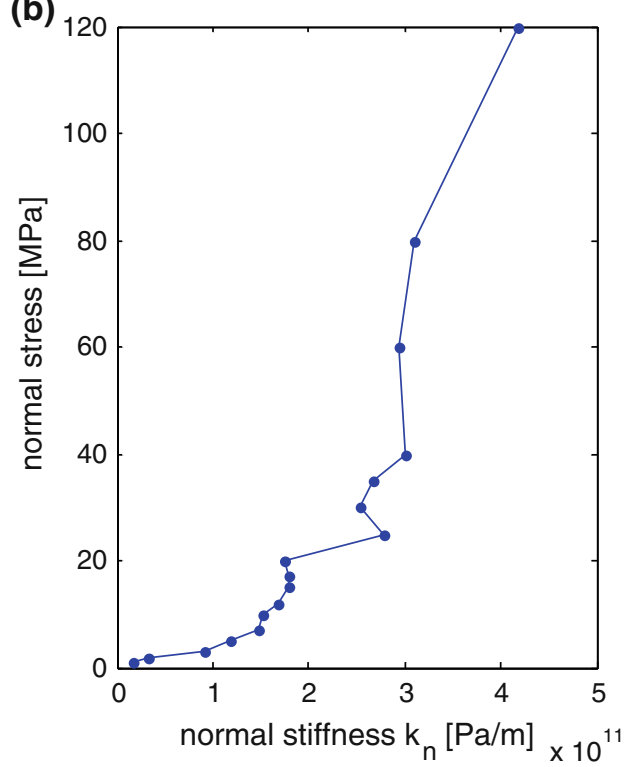

joint surface. Figure 10a shows how contact forces pass from one side of the model to the other one through the active contacts. The visualization of the contact forces is a very good tool to understand the mechanics of joint closure in the model and in real joints, if the model is accurate.

This method for modelling joint closure can still be improved, but already provides a simple method to model joint closure and its main features. The following considerations regarding this model can be made. First, the main mechanisms of joint normal behaviour are correctly represented and; second, though very simple, the method of joint generation resulted in a competent two dimensional representation of planar low roughness joint.

The major shortcomings of the method are (a) complete closure of the joint is attained for very high normal stress, (b) parametric control over the behaviour is difficult (trial and error), (c) real asperities have longer wavelengths and (d) characteristic of undulating fractures are not represented.

\subsection{Stress Wave Propagation in BPM}

To simulate the stress-wave interaction with the fracture it is necessary to understand how waves propagate and are changed while crossing the dense non-organized bonded particle assemblies that are used to simulate the rock material.

The study of the mechanics of particulate media has been object of research for some decades. Mindlin (1954) summarizes some of the first works on a mathematical theory of small deformation of compact granular media. Several other authors contributed to the derivation of stress-strain relations for planar and spatial organized arrangements of disks or spheres. Analytical stress-strain relations and consequently wave velocities for simple particle organization schemes (rectangular, hexagonal and cubic) have been derived (Duffy 1959; Hoover et al. 1974) and point the way in more complex cases and in assessing the performance of particle codes. Mechanical properties of unstructured assemblies or particles with non-uniform diameters cannot be determined analytically. It is only possible to statistically characterize the particle fabric through such quantities as the particle size distribution, particle density or porosity, coordination number and branch vector (Tai and Sadd 1997).

\subsection{Dynamic Properties of Organized and Unorganized Particle Assemblies}

By considering the equivalence between a rectangular or hexagonal constant radius particle organization and a solid that encloses the particles, dynamic properties for both arrangements can be found. In a rectangular packing of constant radius undeformable disks or spheres, each particle has four neighbours while in a hexagonal arrangement each particle is in contact with six other particles. In rectangular packings, there is no particle imbrication, so Young's ratio is null while in hexagonal packings with constant normal and shear stiffness the Poisson's ratio is 0.25 . These are the simplest modes of organizing circular constant radii particles. The porosity or void ratio of rectangular and hexagonal disk assemblies, which yields the relation between the particle assembly and an equivalent continuum density that encompasses it is $1-\pi / 4 \approx$ $21.5 \%$ and $1-\pi / 2 \sqrt{3} \approx 9.31 \%$, respectively. $9.31 \%$ is the minimum porosity attainable in a disk assembly. Compact 
(a)

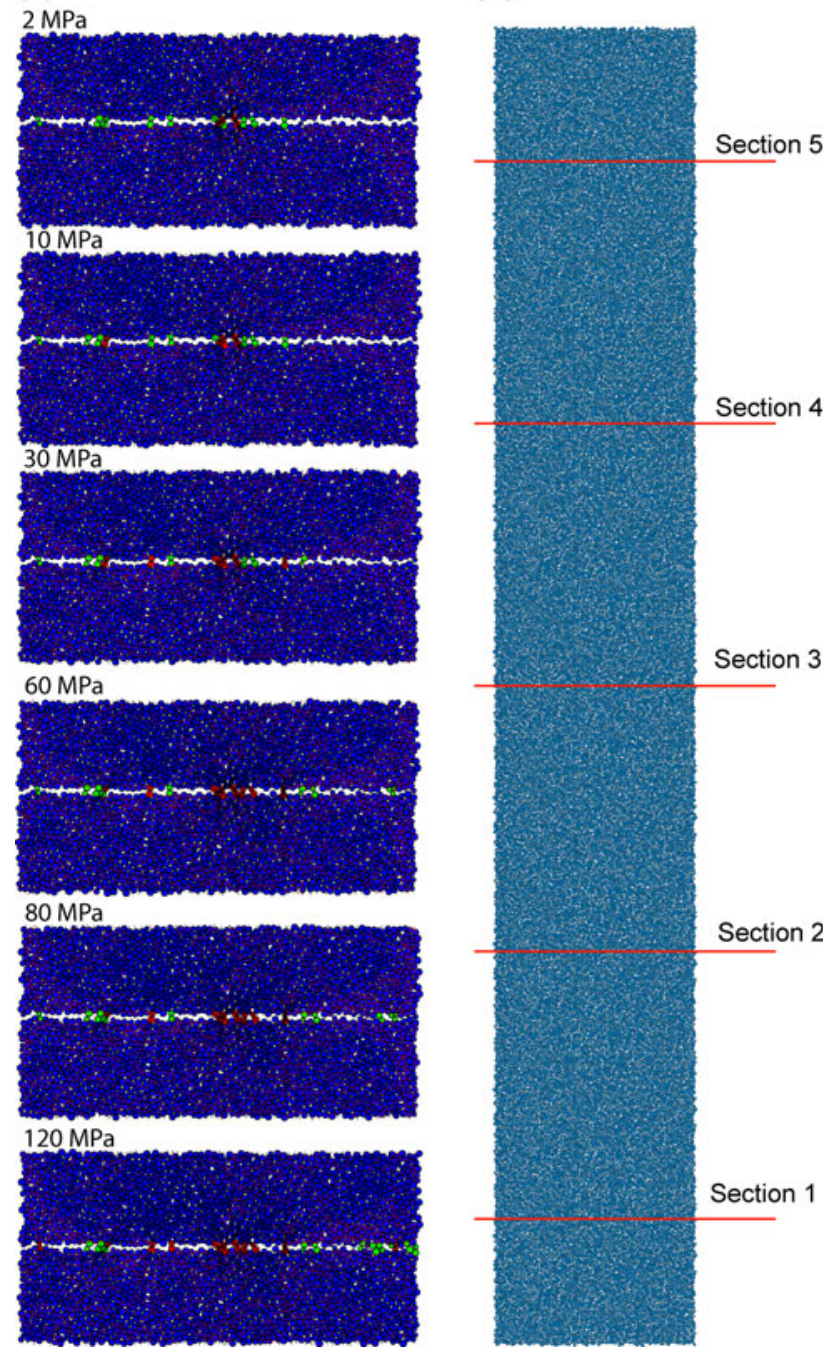

Fig. 10 a Evolution of contacts through the test. Green balls are in contact, red balls are in contact and in the second branch of the constitutive model (Fig. 8). b Sections where balls' velocities are probed in the dynamic part of the study

bonded contact models generated through the method explained above have porosities from 14 to $16 \%$.

Wave velocity for a compressive wave in an array of disks with radius $r$ and thickness $t$ connected with elastic linear springs of stiffness $k_{n}$ is:

$c_{p, \text { disk }}=\sqrt{\frac{4 k_{n}}{\pi \rho_{\text {disk }}}}$.

A shear wave in a string with shear contacts of stiffness $k_{s}$ has equivalent solution. While in static calculations it is indifferent if disk or sphere-like particles are used (given that the self-weight is correctly calculated), in dynamic calculations particle mass influences wave velocity. Equations 9 show that in disk assemblies wave velocity is independent of radius, which does not happen in sphere assemblies, so the former are used.

The solution for a hexagonal packing of disks with no shear interaction $\left(k_{s}=0\right)$ is:

$c_{p, \text { disk }}=\sqrt{\frac{9 k_{n}}{2 \pi \rho_{\text {disk }}}}$ and $c_{s, \text { disk }}=\sqrt{\frac{3 k_{n}}{2 \pi \rho_{\text {disk }}}}$.

If the particle arrangement is not organized due to particle disposition irregular size and/or properties distribution, analytical solution is not possible and wave velocity must be found by testing each model.

\subsection{From Static to Dynamic Analyses}

Dynamic analyses are frequently preceded by static calculations where an initial stress state may be found. This is done too in the present study. In the static calculation, the top half of the model is loaded against the bottom half compressing the fracture. In DEM, static equilibrium is reached through the dynamic relaxation method (Belytschko 1983) and the mass of each particle is scaled up or down so that the critical timestep of the model becomes one (a process called density scaling used to optimize runtimes). When the static calculation ends the model is almost at rest since unbalanced forces are small compared with the repulsive forces between particles. As density scaling is turned off, masses return to their real value which is usually several orders of magnitude lower and the same happens to the timestep. However, since the timestep decreases proportionally to the square root of the particles mass, the acceleration of the particles increases generating abnormal particle velocities that compromise the previously attained equilibrium. To prevent this, the timestep must be lowered gradually to allow artificial damping to dissipate the excessive velocities. When this stabilization is attained the dynamic mode is switched on, which corresponds to assigning the rock's correct dynamic damping coefficient turning off the mass scaling and using the correct dynamic timestep (Potyondy DO, 2008, Personal Communication). The second step of the calculation, which consists in the injection of a velocity time-history at the base of the model while the model is kept under the stress of the previous step, may then take place.

\subsection{Dynamic Loadings and Boundary Conditions}

Mechanical loadings or more generally boundary conditions can be kinematic, static, or mixed. Kinematic loadings are materialized by prescription of particle movement (translational and rotational displacement, velocity or acceleration), while static loadings correspond to application of forces and/or moments. 
The correspondence between velocity and stress in continuous media is given by:

$\sigma=\rho \cdot c \cdot \dot{u}_{\text {load }}$

where $\sigma$ is the applied stress, $\rho$ and $c$ the medium's density and wave velocity, respectively, and $\dot{u}_{\text {load }}$ is the load prescribed velocity.

Tests with both velocity and force loadings in $\mathrm{PFC}^{2 \mathrm{D}}$ show that the methods produce practically equivalent waves, the only difference being that the force load occurs with a lag of one timestep as the force load is integrated in the motion equation one timestep after introduction, while an imposed displacement produces its effect immediately.

When a particle's movement is constrained by a velocity loading (in all or just in some degrees of freedom), the corresponding equations of motion are not solved, therefore static boundary conditions cannot be applied. In addition, as the movement at the boundary is constrained it will reflect and invert any incoming wave, which may be inconvenient. A possible solution is to release the boundary balls as soon as the injection of the dynamic load ends. However, waves coming from other sources or waves reflected in the interior of the model may arrive at the border before the end of the loading (Fan et al. 2004 paper discusses in detail the implications of these options). To avoid this kind of interferences and ease the extraction of waveforms from the model, a mixed boundary condition, in which static and dynamic loadings coexist with an absorbing boundary, is implemented in $\mathrm{PFC}^{2 \mathrm{D}}$ via a FISH function.

Absorbing or silent boundaries simulate the dynamic continuation of the domain without representing it. Belytschko (1983) lists some of the most relevant strategies to implement silent boundaries. Of these, viscous boundaries as proposed by Lysmer and Kuhlemeyer (1969) are one of the most widely used strategies. They have been implemented in the source code of all Itasca programs (except $\mathrm{PFC}^{2 \mathrm{D}}$ and $\mathrm{PFC}^{3 \mathrm{D}}$ ) and also on several popular FEM codes.

When a wave hits a viscous boundary, a symmetric wave that cancels the incoming one is generated. This symmetric wave is materialized by imposition of equivalent forces such as in the velocity-stress Eq. 10. A minus sign and $\dot{u}_{\text {body }}$, standing for the velocity in the interior of the model are included:

$\sigma=-\rho \cdot c \cdot \dot{u}_{\text {body }}$.

Forces calculated through this equation are added to other forces (contact forces, external static or dynamic loads, gravity, etc.) acting at that particle. This allows the edge to simultaneously support an absorbing boundary, and static and dynamic loading. The cancelling wave is produced with a delay of one timestep, but this lag is usually too small to introduce errors. The density of the boundary particles must be set to half of its real value, in order to simulate the continuation of the domain. If a dynamic load is to be introduced simultaneously in the same place of an absorbing boundary, it must be set as a force loading. Because half of the load will be absorbed by the viscous boundary, the load magnitude must be doubled.

To translate dynamic stress into particle forces, particle dimensions and boundary depth must be taken into account via the boundary depth correction factor $b f_{\text {depth }}$ displayed in Eq. 5.

$$
\begin{aligned}
F_{\text {ball }} & =b f_{\text {depth }} \cdot \sigma \cdot A_{\text {ball }} \\
& =\frac{l_{\text {bound }}}{2 \cdot r_{\text {mean }} \cdot n b_{\text {bound }}} \cdot \rho \cdot c \cdot u_{\text {load }} \cdot 2 \cdot r_{\text {ball }} .
\end{aligned}
$$

In the top boundary of the model this results in

$$
\begin{aligned}
& F_{\text {ball,load }}=\frac{69}{164} \cdot \rho \cdot c \cdot u_{\text {load }} \cdot 2 \cdot r_{\text {ball }} \text { and } \\
& F_{\text {ball, absorb. bound. }}=-\frac{69}{164} \cdot \rho \cdot c \cdot \dot{u}_{\text {ball }} \cdot 2 \cdot r_{\text {ball }}
\end{aligned}
$$

for dynamic loads and viscous boundaries.

Another particularity in the application of viscous boundaries in unorganized particulate media is that shear or compressive wave velocity are not available prior to the calculation, they must be measured in a previous run.

The hybrid boundary is implemented in PFC and tested with pure shear and compressive waves hitting linear boundaries at normal angles. They presented an efficiency (defined as the ratio between reflected and incoming wave amplitude) superior to $99 \%$.

It is well known that viscous absorbing boundaries loose efficiency as the angle of incidence decreases. Further tests are necessary to investigate these limits in particulate media. We must take into consideration that the response of the absorbing boundaries under mixed shear and compressive waves has not been verified. This step is also essential because both kinds of waves are present in most real cases.

\subsection{Load Waveform}

The ultimate goal of this study is to mimic the passage of dynamic compressive waves across fractures, so a wave of frequency content similar to the studies of wave-fracture interaction in real joints is used (Pyrak-Nolte et al. 1990; Cai 2001). The Ricker wavelet is a null phase function frequently employed in geophysics to model seismic waves. It is the normalized second derivative of a Gaussian function, and a special case of the family of continuous wavelets known as Hermitian wavelets. It is composed by one positive central peak surrounded by two symmetric 
lateral lobes, as represented in Fig. 11. The mathematical expression of a Ricker wavelet is

$v(t)=\left(1-2 \pi^{2} f^{2} t^{2}\right) \mathrm{e}^{-2 \pi^{2} f^{2} t^{2}}$

where $f$ is the central frequency, the only parameter of the wave equation, and $t$ is time. The function shows no high frequency corners that aggravate wave dispersion and the first frequency component is zero. The plot of the absolute value of the Fourier transform is not symmetric, vanishing when frequency approaches zero and when the maximum frequency, which is approximately three times the central frequency, is reached. The complete duration of a Ricker wave corresponds to a frequency that is around $2 / 3$ of the peak frequency expressed in the wave function $(6,667 \mathrm{~Hz}$ in Fig. 11).

\subsection{Quality of Wave Propagation}

Since the discrete numerical representation of a wave never expresses with all detail the original continuous waveform, waves propagating in a numerical model inevitably have their characteristics degraded. The principle behind wave transmission quality is that the shortest wavelength of interest must be correctly represented. Quality requirements vary with the modelling situation. If only the wave arrivals are needed, more degradation can be allowed but if peak amplitude or the entire waveform are to be used quality requirements increase. To adjust ball's radius to maintain the wave quality across the model can result in excessively heavy models. To prevent this, the following strategies may be used: (a) the quality of the wave transmission must be partially sacrificed taking into account the minimum necessary degree of precision, (b) the size of the model is reduced (employing absorbing boundaries) or (c) the frequency of the signals is scaled down or filtered to eliminate the highest frequencies if their energy content is not significant.

Toomey (2001) derived dispersion laws for hexagonal (hence, constant radius) arrangements of spheres. An equivalent expression can easily be deducted for disk models but it is still of limited value when working with unorganized BPM. Hazzard and Young (2004) studied the consequences of thermal and mechanically induced cracking in the seismic velocity in brittle rock. Since they were interested in measuring wave arrival times they did not focus on the preservation of wave characteristics. In this work, however, not only the wave arrival times but also the whole waveform is necessary. It is also desirable that the effect of the "continuum" part of the model on the wave is as small as possible to isolate the effect of the
Fig. 11 Ricker wavelet with central frequency of $10^{4} \mathrm{~Hz}$ and unit amplitude shifted to the positive time domain. Velocity (a), displacement (b) and absolute value of the velocity FFT (c) (a)

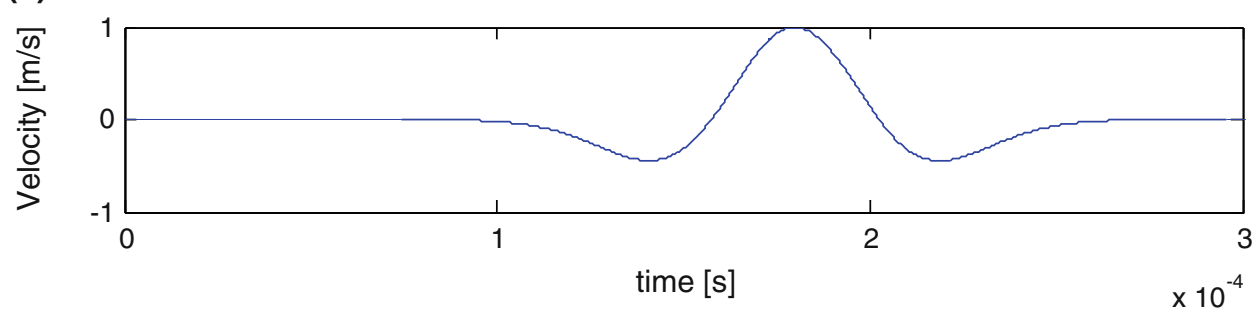

(b)
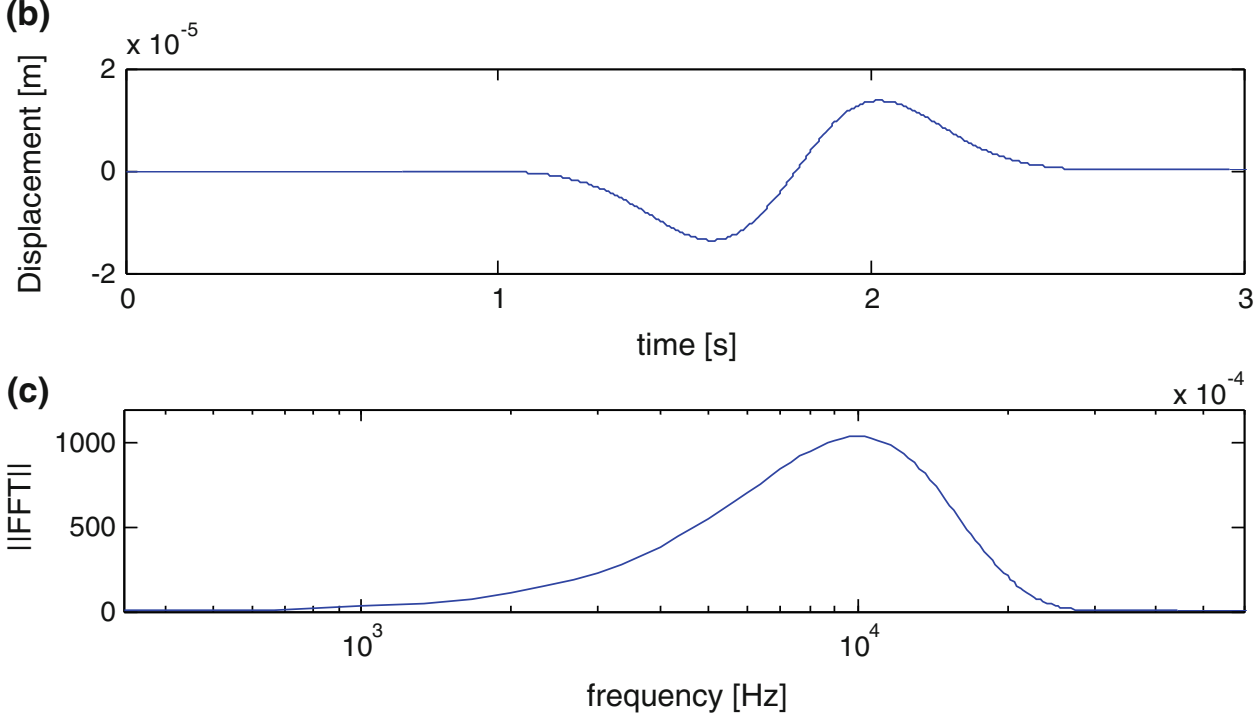
fracture. The parameter chosen to characterize wave quality in dense non-structured particle assemblies is the wavelength over the average ball diameter.

To determine the minimum number of particles per wavelength in particle models, Ricker waves with central frequencies from 5 to $200 \mathrm{kHz}$ are injected at the base of the model. Figure 10b presents the model and the sections where waves are probed. These sections are 6, 180, 300, 420 and $450 \mathrm{~mm}$ from the bottom. A single particle is hardly representative of the behaviour of a section, as it can be in one extreme of radii distribution, less connected with its neighbours or outside of the local path of wave passage. This is especially true after a singularity such as a discontinuity or a void. Five particles, distributed horizontally across the model, are probed in each section and their arithmetic average is calculated.

Several BPM models generated using the same seed parameters were tested and the very small variation in dynamic properties is confirmed. Other methods of generation of compact packings may result in slightly different particle organization, but if the radii distribution porosity and contact type are similar this study's conclusions should hold valid.

In order to compare and frame the dynamic response of the BPM, three other assembly types with the same external dimensions, balls radius equal to the average radius of the BPM model and similar microproperties where generated. Ball density that plays an important role in the definition of dynamic properties is set to a value so that all assemblies have an equivalent continuum density of $2,700 \mathrm{~kg} / \mathrm{m}^{3}$. Ball and contact properties are displayed in Table 1.

In the rectangular model, contact normal stiffness is set equal to the normal stiffness in the BPM model. The same value is attributed to normal and shear contacts in the first hexagonal assembly model $\left(\operatorname{Hexa}_{k n=k s}\right)$, while in the second hexagonal model $\left(\mathrm{Hexa}_{k n=2.5 \mathrm{ks}}\right)$ normal stiffness is two and a half times the shear stiffness, this being the organized packing that resembles more the BPM. Similar static and dynamic boundary conditions are applied in all models. Absorbing boundary conditions are applied to the top of the model and the waves are injected at the bottom by prescribing a velocity time-history. Movement in the direction normal to the wave propagation is restricted in lateral boundaries. This simulates a semi-infinite medium in this direction thus preventing the generation of surface waves that drain energy from the compressive wave.

Timestep is set to $5 \times 10^{-8} \mathrm{~s}$ that is slightly smaller than the maximum stable timestep in all models. No damping is used because all alterations to the waveform must come from assembly-caused dispersion. Waves with central frequencies of $5,10,20,30,40,80,120,150$ and $200 \mathrm{kHz}$ were tested in each of the four models.

\subsection{Wave Velocity}

Wave propagation velocity is calculated using the time lapse between the wave arrivals in the five sections of the model shown in Fig. 10. Figure 12 displays the velocity of propagation measured in several sections for all tested frequencies. Wave velocity is fairly constant in the central and final areas of the model where the wave has stabilized its progression mode.

At the bottom of the model where the velocity loading is imposed, the wave has to adapt to the particulate medium as it "chooses" local paths of progression. The effect is stronger in the unorganized packing and when number of balls per wavelength is lower. Notwithstanding the variation in wave velocity in the initial zone of the BPM and $\mathrm{Hexa}_{k n=k s}$, the velocity values measured in the middle sections of each model are stable. These are the propagation velocity values used in the subsequent analyses. Figure 13 displays the relative evolution of propagation velocity with wave frequency. It can be seen that wave velocity is almost constant across the considered frequency range and that higher frequencies travel at slightly higher velocities as is characteristic of dispersive media. It should be noted that even for the higher frequencies when the waveform is almost completely destroyed, wave velocity is nearly constant showing that wave velocity is independent of wave quality.

With a non-organized structure, BPM is the most dispersive assembly: from 5 to $200 \mathrm{kHz}$, wave propagation velocity increases around $3 \%$. The variation is smaller in the other assemblies, topping at about $1 \%$. Given that $c_{p}$ presents small variation with frequency in the range considered, an average value is used in the subsequent studies. Compressive wave velocities are then $4,191 \mathrm{~m} / \mathrm{s}$ in the

Table 1 Ball and contact properties in the four modelled packings

\begin{tabular}{lllllll}
\hline & Radius $(\mathrm{mm})$ & $k_{n}(\mathrm{GN} / \mathrm{m})$ & $k_{s}(\mathrm{GN} / \mathrm{m})$ & $\rho_{\text {ball }}\left(\mathrm{kg} / \mathrm{m}^{3}\right)$ & Porosity $(\%)$ & $\rho_{\text {continuous }}\left(\mathrm{kg} / \mathrm{m}^{3}\right)$ \\
\hline BPM & $0.5-0.83$ & 62.0 & 24.8 & 3,150 & 14.3 & 2,700 \\
Rectangular & 0.665 & 62.0 & - & 3,438 & 21.5 & 2,700 \\
Hexa $_{k n=k s}$ & 0.665 & 62.0 & 62.0 & 2,977 & 9.31 & 2,700 \\
Hexa $_{k n=2.5 k s}$ & 0.665 & 62.0 & 24.8 & 2,977 & 9.31 & 2,700 \\
\hline
\end{tabular}


Fig. 12 Propagation velocity $c_{p}$ along the model for all tested frequencies (legend shows peak frequency of the waves, in $\mathrm{kHz}$ )

Fig. 13 Relative variation of compressive wave velocity with peak frequency
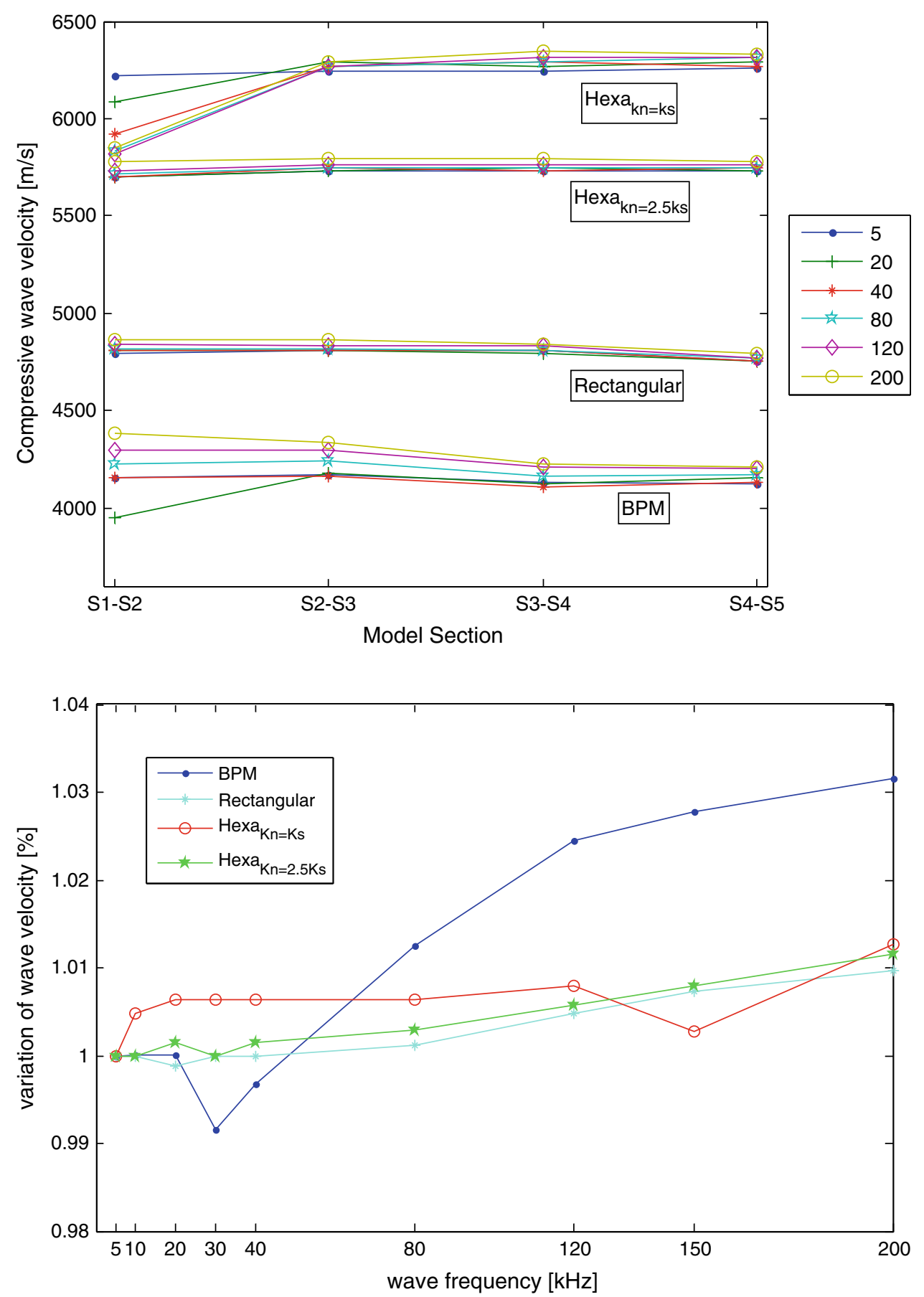

BPM assembly, $4,819 \mathrm{~m} / \mathrm{s}$ in the Rectangular assembly, $6,279 \mathrm{~m} / \mathrm{s}$ in Hexa $_{k n=k s}$ assembly and $5,750 \mathrm{~m} / \mathrm{s}$ in $\mathrm{Hexa}_{k n=2.5 k s}$ assembly. The lower wave velocity occurs in the BPM, which is logical because this is the packing with the lowest degree of organization and waves have to find and follow less direct paths to travel across the model. Between the two hexagonal packings there is a $10 \%$ difference in wave velocities as the smaller shear stiffness in the $\mathrm{Hexa}_{k n=2.5 \mathrm{ks}}$ model cuts wave velocity. Since waves travel with different velocities in the various models, waves with equal frequencies will have distinct lengths.

\subsection{Wave Attenuation}

The other key parameter in wave propagation in numerical models is the way in which the quality of the wave representation as it crosses the model. To assess this, waves of increasing frequencies are sent across the model and the 
peak amplitude recorded at the sections indicated in Fig. 10b. The decay of peak amplitude is chosen to be the quantitative indicator of wave quality because it has been observed that waveform degradation is proportional to decrease in the maximum amplitude. In Fig. 14, the relative amplitude decay is plotted against distance divided by the average ball diameter. The initial amplitude value is measured at Sect. 1 (Fig. 10b) placed near the base is the reference value. In all organized assemblies, the rate of amplitude loss is constant meaning that wave degradation accumulates while the wave travels. In the BPM model, degradation is higher at the beginning of the model where the wave adapts to the particulate medium. After that initial adjustment, the rate of peak loss is approximately constant.

Figure 15 provides a compact convenient way to compare the performance of all models, representing the relative loss of velocity amplitude between the end and the start of the model for all studied wavelength-ball diameter relations. It should be noted that only in the rectangular packing particles are perfectly aligned with the particle movement thus only in this case the wavelength/ball diameter ratio is absolutely meaningful.
If a maximum loss of amplitude of $5 \%$ is set, the wave frequency and associated wavelength/particle diameter relation that complies is 82 for the BPM, 24 for the Rectangular, 31 for the $\operatorname{Hexa}_{k n=k s}$ and 42 for the $\mathrm{Hexa}_{k n=2.5 \mathrm{ks}}$. BPM model is much more demanding than all organized packings. Of these, the one that preserves better wave quality is the rectangular packing, followed by the Hexa $_{k n=k s}$ and Hexa ${ }_{k n=2.5 k s}$.

\section{Propagation of Compressive Stress Waves Across Fractures in BPM}

Wave-fracture interaction numerical tests come in the sequence of the static joint closure tests and are possible because of the knowledge on wave propagation gained in the previous section.

\subsection{Numerical Tests Description}

At the end of each load increment of the joint compression calculation, the model is saved. In some selected cases
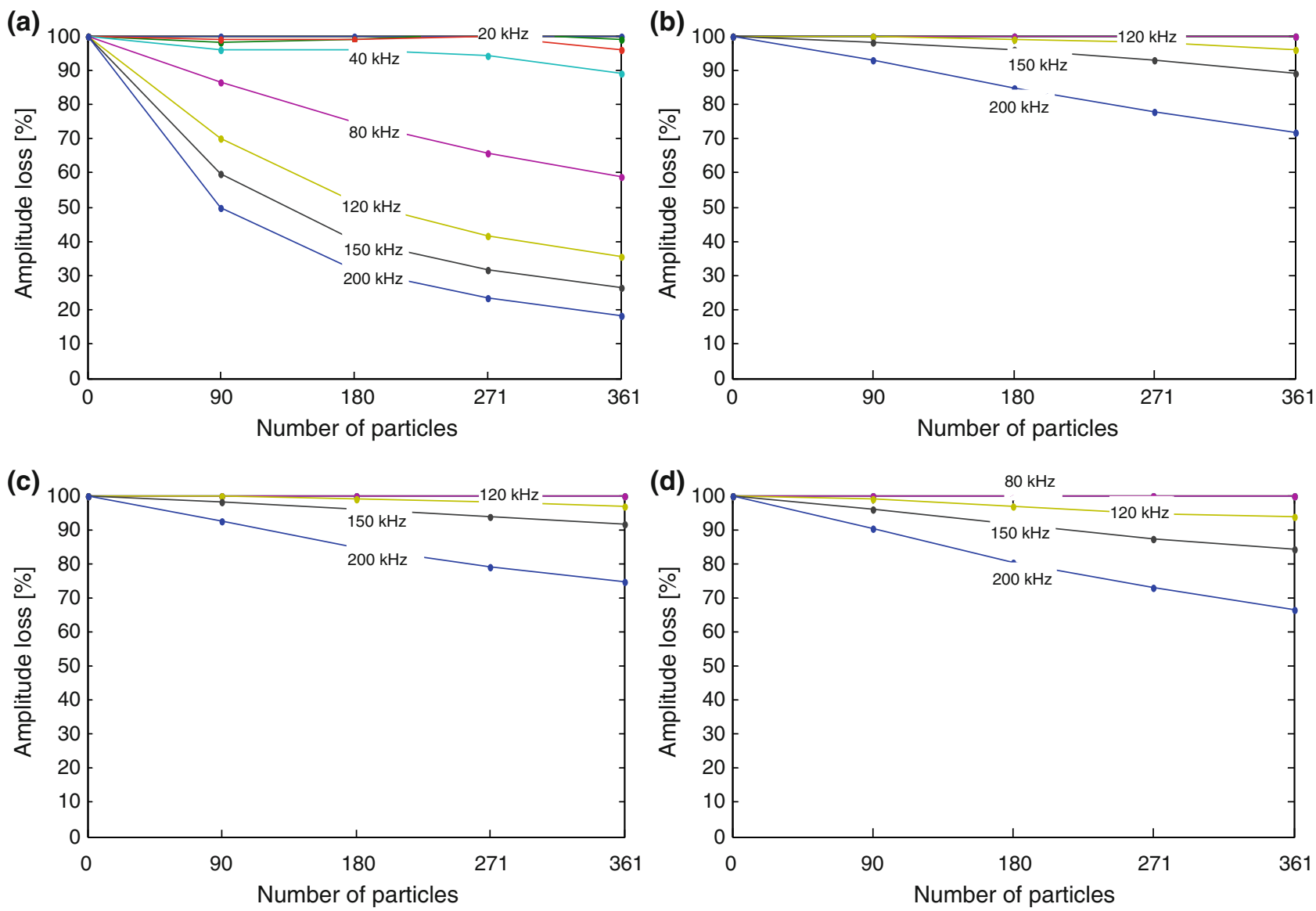

Fig. 14 Relative variation of peak wave amplitude along the models length for all tested frequencies: BPM (a), Rectangular (b), Hexa $k_{k n=k s}(\mathbf{c})$ and $\operatorname{Hexa}_{k n=2.5 \mathrm{ks}}(\mathbf{d})$. Legend shows peak frequency of the waves 
Fig. 15 Relative variation of peak wave velocity measured at the bottom (Sect. 1) and at the top end (Sect. 5) of the model. Plot (b) zooms in the axes origin and horizontal line marks $5 \%$ loss
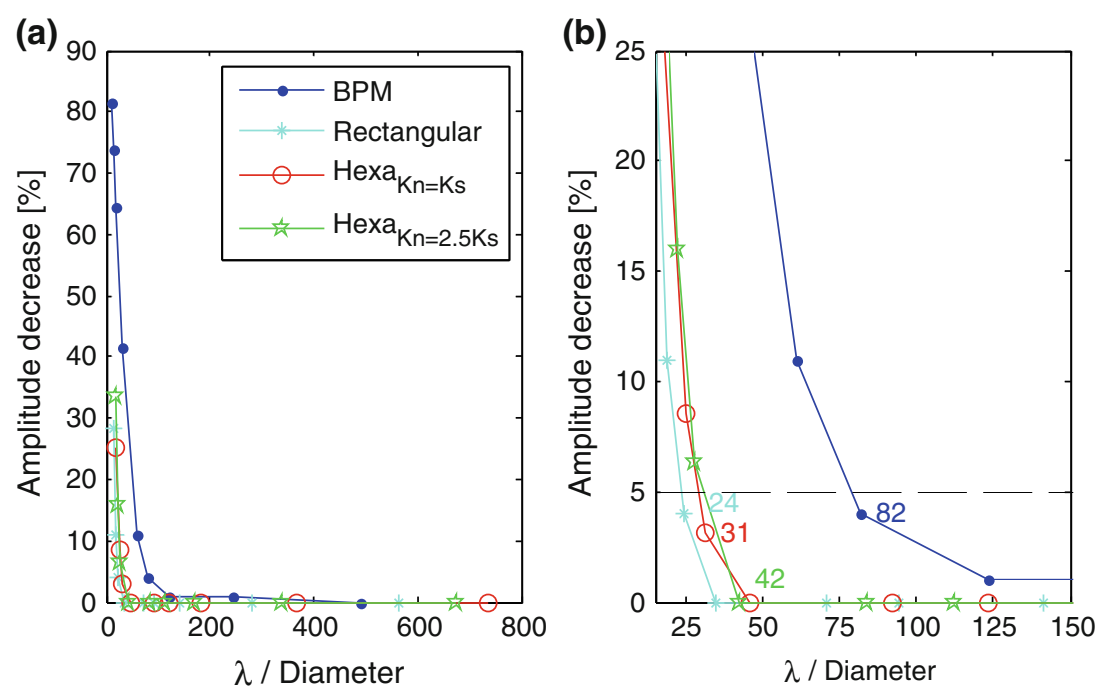

(compression of 1, 5, 17.5, 25, 80 and $120 \mathrm{MPa}$ ), a Ricker wave with a peak frequency of $15 \mathrm{kHz}$ is injected at the bottom boundary. The input wave, the wave that crosses the fracture (Transmitted) and the wave that is bounced back (Reflected) are processed and the dynamic characteristics of the fractures are calculated according to the DDT (Pyrak-Nolte et al. 1990).

In the joint closure tests, a vertical downwards force corresponding to the normal stress is installed in the balls at the top boundary. This force is maintained and an absorbing boundary is installed at this location. The balls velocity at the bottom boundary is constrained and kept null except for the injection of the Ricker wave history loading. Horizontal movement at the lateral boundaries is constrained to prevent formation of surface waves. No damping is used and timestep is set to $5 \times 10^{-8} \mathrm{~s}$, below the minimum stable timestep.

Particle velocities are recorded at three sections located at the bottom and top of the model and $135 \mathrm{~mm}$ (one and a half times the width) below the fracture located at midheight. At each section, five balls are probed. The input wave is extracted from the section nearer to the bottom and the transmitted wave from the section at the top border. The reflected wave is captured at the section below the fracture. As the upwards-going input and downwardstravelling reflected waves superimpose in time it is necessary to isolate the reflected wave by subtracting the input wave to the total recorded waveform. The section where the transmitted wave is recorded is chosen at a distance from the fracture that allows the reconstruction of the wave after the passage through the fracture since during the passage through the fracture the wave "squeezes" through the active contacts, as illustrated in Fig. 16. One of the rewards of micromechanical models is exactly the visualization of this kind of fast microscopic phenomenon.

\section{Results and Discussion}

According to the DDT, the analysis of the waves that originate from a rock fracture when it is excited by an impinging wave allows, the identification of the dynamic, or seismic, stiffness of the fracture. It is expected that the more the fracture is compressed and closed the better it will transmit the wave to the other side and the less it will reflect.

Since the input wave frequency content and the intact material impedance are known, it is possible to find a dynamic stiffness that generates $T(\omega)$ and $R(\omega)$ curves that adjust the ones measured in the model. In laboratory tests of rock fractures (Cai 2001; Pyrak-Nolte et al. 1990, among others), a good adjustment between measured and theoretical wave amplitudes has been registered. Can the numerical model of a fracture that mimics the microscopic deformation mechanics do the same?

Figure 17 shows how the waveforms of the reflected and transmitted waves evolve while the compression in the joint increases. A minimum treatment of the time series is necessary to isolate the reflected and transmitted wave from other waves that pass the measurement spot simultaneously. A tapered cosine time-window with a tapper of 5\% (Achenbach 1973) is applied in the time domain to the series of the reflected wave. When the joint is lightly compressed, the transmitted wave is nearly imperceptible and the reflected wave almost equals the input wave. With increasing compression, the transmitted wave grows and the reflected wave shrinks. This can be observed in the absolute value of the Fourier transform of the input and of each of the transmitted and reflected waves.

Since wave passage produces a reflected and a transmitted wave, two seismic stiffness values can be determined. This is an advantage in relation to the laboratory 
Fig. 16 Ball velocity vectors of wave passing across fracture. a Vectors and balls, b Velocity vectors only (a)

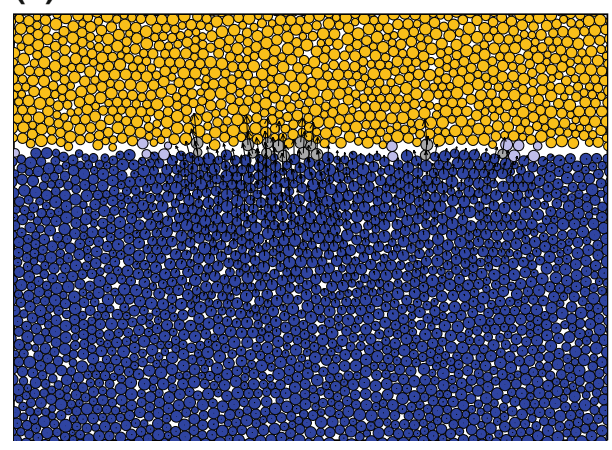

(b)

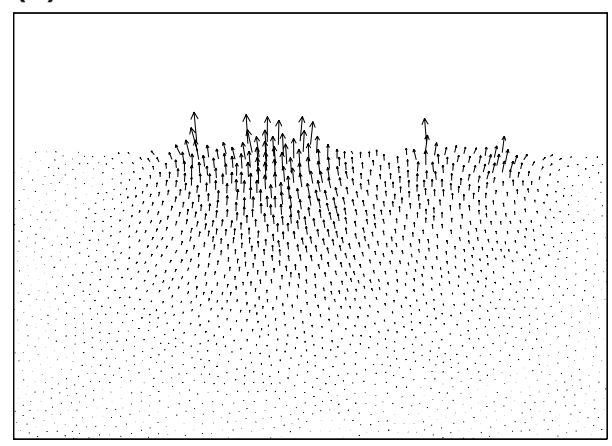

Fig. 17 Input wave is the first positive peak (in blue), reflected wave is the second positive peak (in red) and the transmitted wave is the third positive peak (in green)

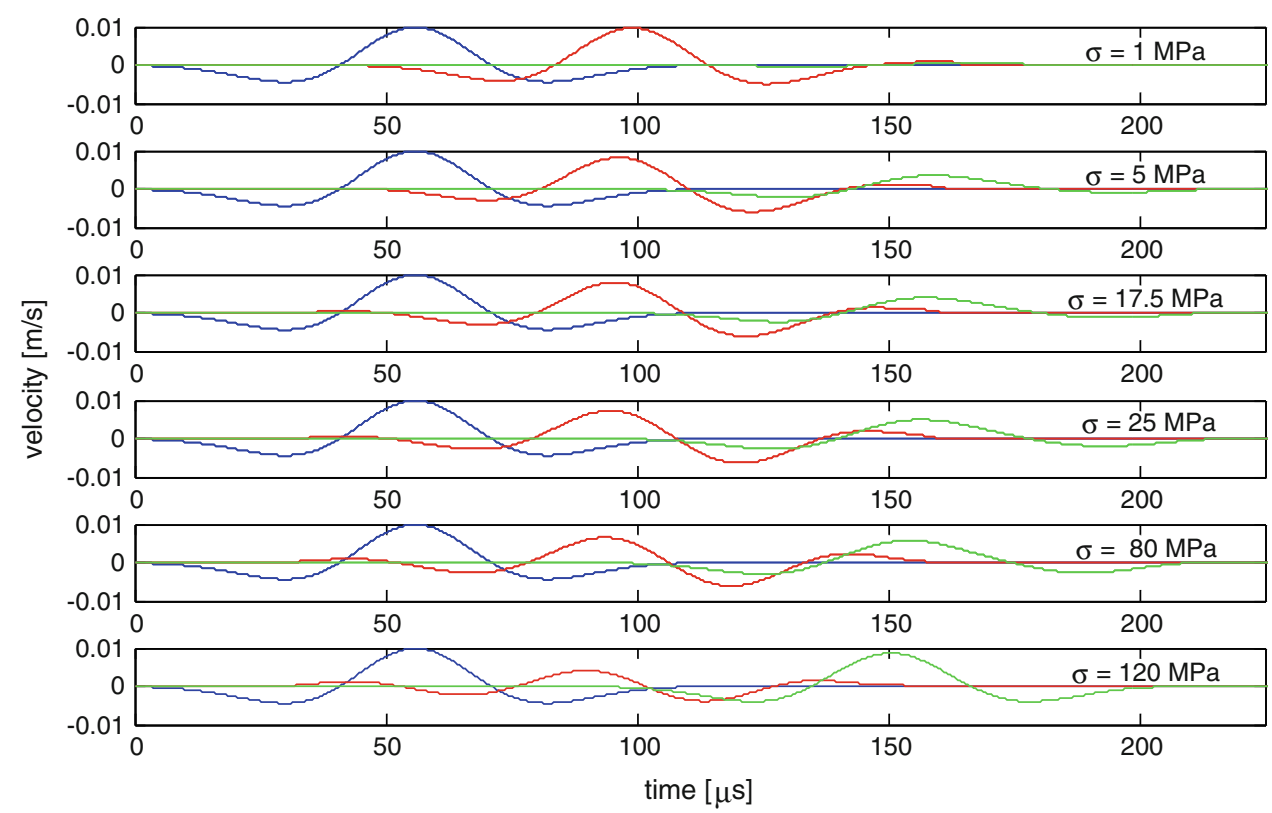

tests found in the literature where only the transmitted waves are measured.

Figures 18 and 19 show the fit between the model's reflected and transmitted frequency amplitudes and the DDT predictions. To find DDT's predicted transmitted and reflected waves, optimal dynamic stiffness values $\left(K_{R}\right.$ and $\mathrm{K}_{\mathrm{T}}$ ) must be determined. In an initial step, Eqs. 1a and $b$ are fed with the incident wave $I(\omega)$ and with tentative stiffness values. The resulting frequency amplitudes are compared with the models' and new stiffness values are tried until an optimum fit of the curves is attained.

This fit is better in transmitted waves, which can eventually be explained by the fact that these are not contaminated by the incident wave. Both model and predicted reflected waves' central frequency increase with compression stress, but this effect is stronger in the model, causing the adjustment to degrade in higher stresses. As a whole model and predicted transmitted waves match up very well, the correspondence being almost perfect in lower frequencies. In higher frequencies, the model amplitude is slightly higher. This divergence can be attributed to the hypothesis that, contrary to what is admitted in the DDT, dynamic stiffness is not constant being a function of frequency. Pyrak-Nolte and Nolte (1992) investigated this hypothesis and attributed the variation of the stiffness with the frequency to the relation of the wavelength to the different spacing between contact points. To test this hypothesis, simulations where the effect of the average spacing between contact points on dynamic stiffness is studied are necessary.

As predicted by the DDT and observed in laboratory tests, the fracture acts as a low-pass filter transmitting preferentially the lower frequencies; and this effect is stronger in the lower normal stresses when the seismic stiffness is lower and the ratio $\omega \cdot Z_{p} / K_{n}^{\mathrm{dyn}}$ is higher. Even at the maximum stress of $120 \mathrm{MPa}$, the wave is not completely transmitted and the reflected wave is still visible meaning that the fracture is not totally closed. The 
Fig. 18 Frequency content of the reflected waves (in red) and DDT prediction (in black). Input wave (in dashed blue) is shown for reference
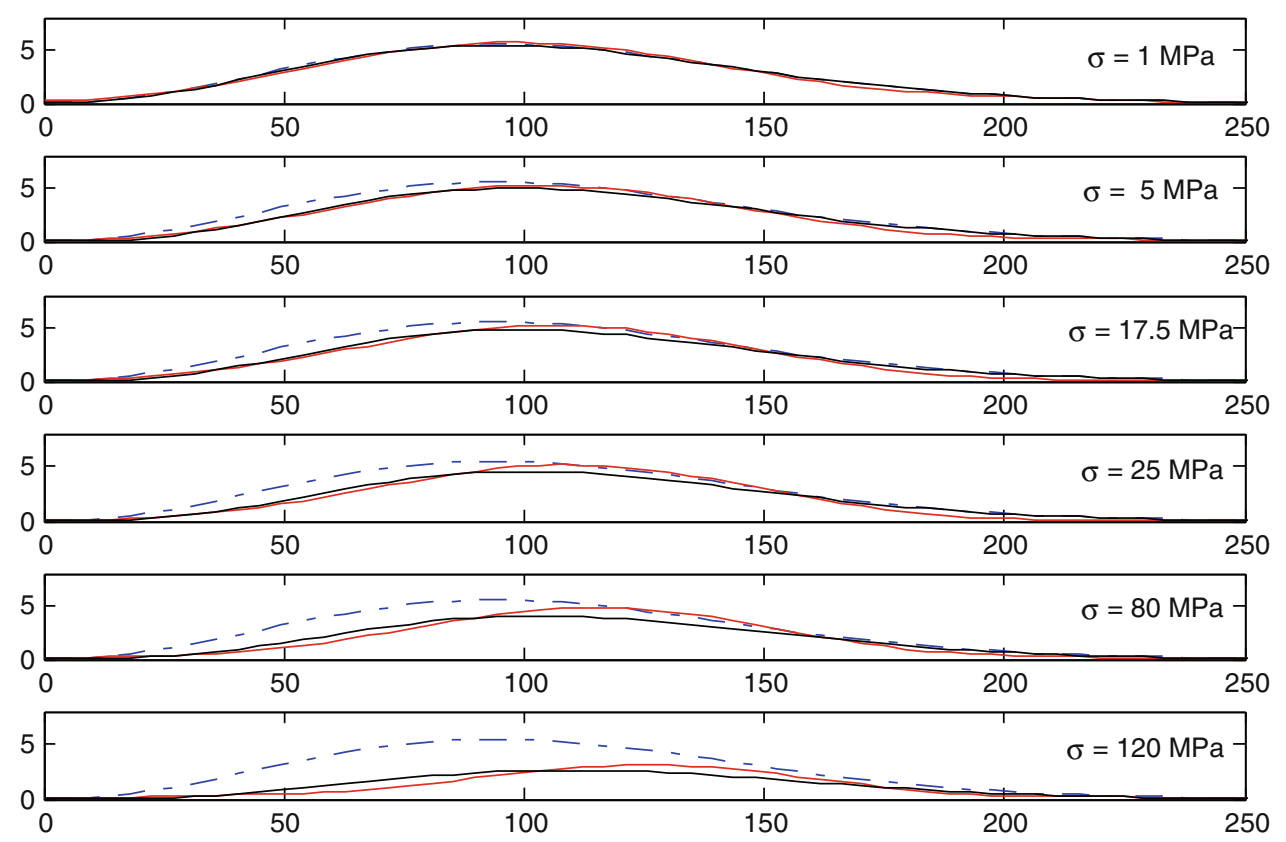

angular frequency $\left[\times 10^{3} 2 \pi / s\right]$

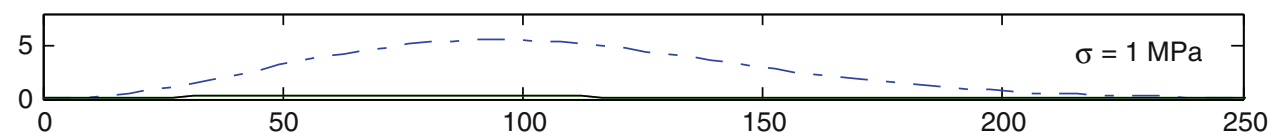

the transmitted waves (in green) and DDT prediction (in black). Input wave (in dashed blue) is shown for reference
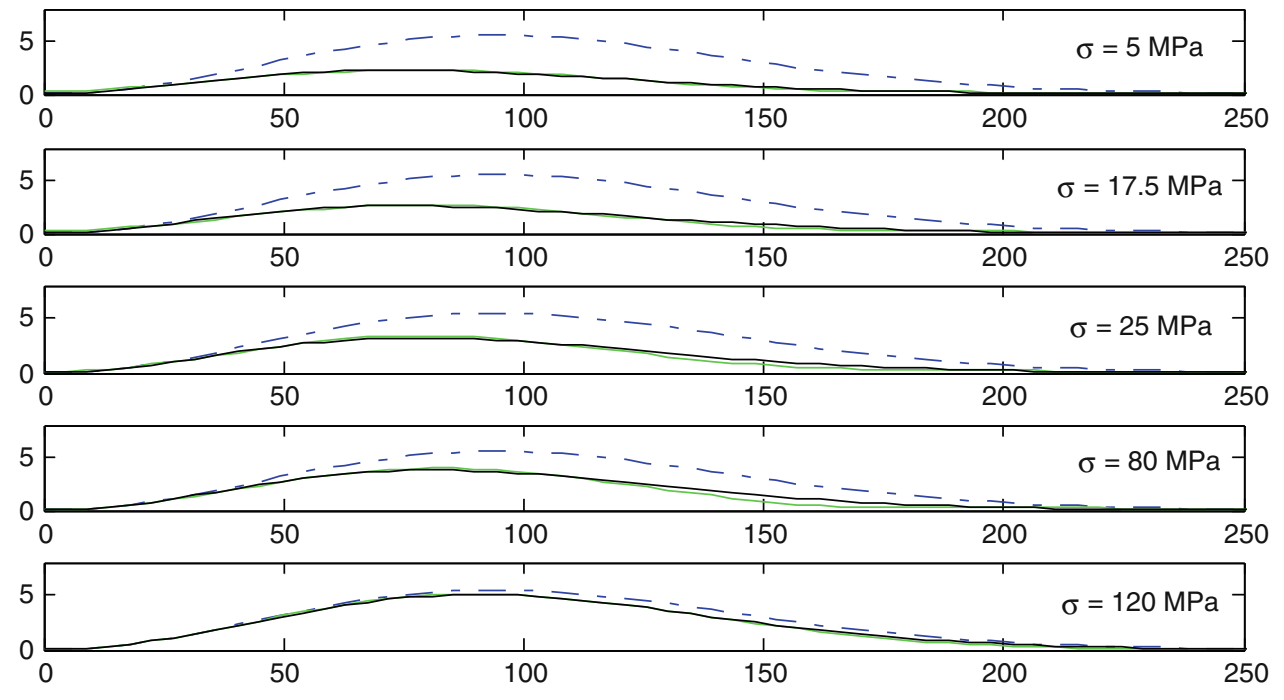

angular frequency $\left[\times 10^{3} 2 \pi / s\right]$

stress-displacement curve of the static joint compression test is also not completely vertical at the maximum stress level.

The fracture behaves elastically: the wave does not loose energy when crossing it $\left(\sqrt{R^{2}+T^{2}}=1\right)$. Figure 20 plots the transmission and reflection coefficients and the square root of their sum, their sum, which is very close to one, corroborates the energy conservation. The only exception is the test under the lowest stress $(1 \mathrm{MPa})$ where the transmitted wave is nearly flat and the reflected wave is almost equal to the input wave. In this scenario, the error margin of the fitting routine that finds stiffness values adjusting theoretical and measured frequency curves is considerable.

Displacement Discontinuous Theory and common sense indicate that the dynamic stiffness should grow with the 
Fig. 20 Reflection (red) and transmission (green) coefficients and energy balance (black)

Fig. 21 Evolution of the dynamic and static tangent stiffness with normal stress. Dynamic stiffness are obtained from analysis of reflected and transmitted waves $\left(k_{\mathrm{R}}\right.$ and $\left.k_{\mathrm{T}}\right)$, static stiffness $k_{\text {static }}$ is extracted from the stress-displacement graph (Fig. 9b)
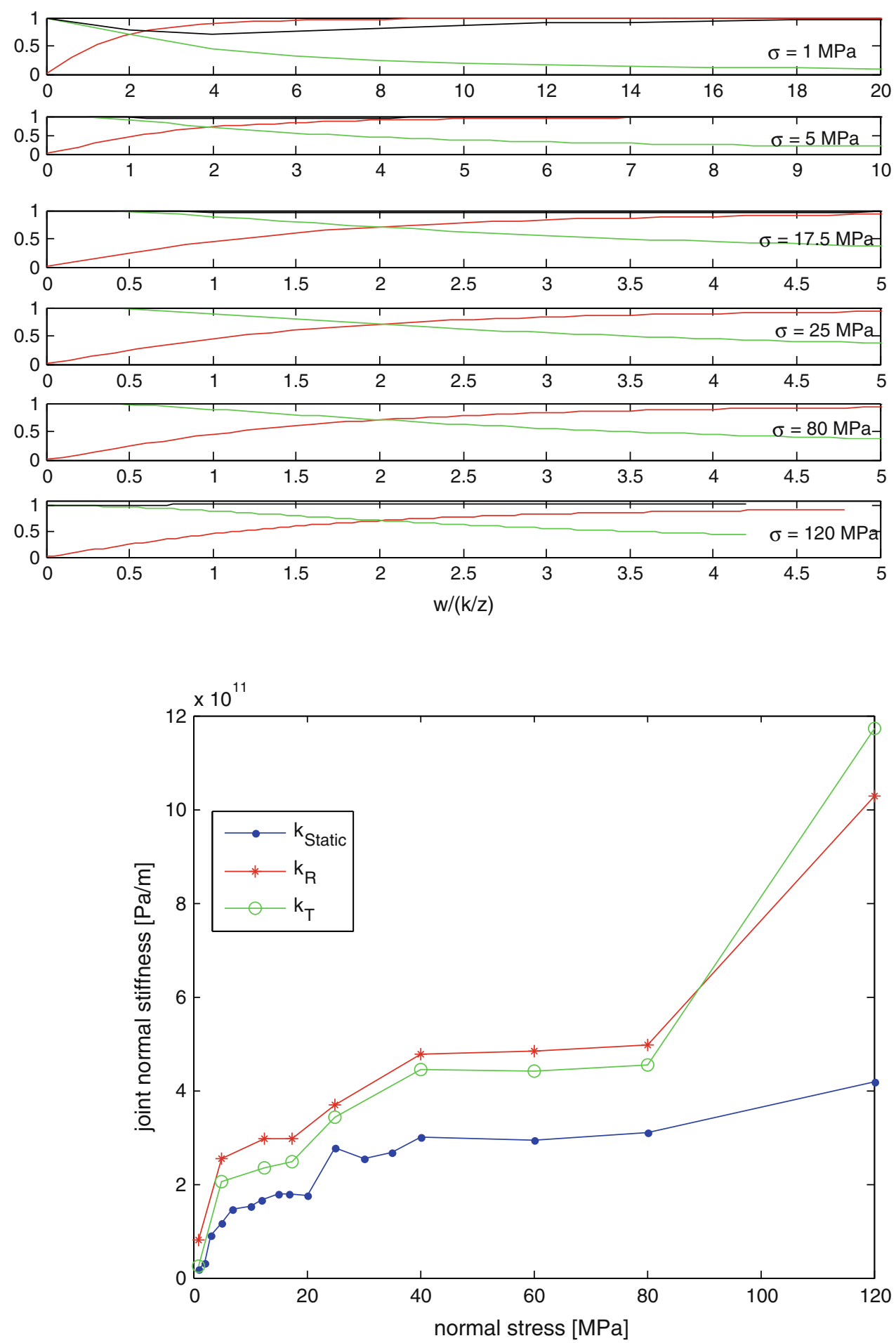

joint normal stress. The evolution of the transmitted and reflected dynamic stiffness with static normal stress is similar (Fig. 21) and the small gap between them is thought to be due to the better quality of the transmitted wave. The energy balance and the match between both stiffness values demonstrate that the BPM joint correctly simulates the dynamic behaviour of rock joints. Figure 21 also displays the evolution of static stiffness in the same joint. The trend followed by the static and seismic stiffnesses, both being tangent values, is similar with the latter being always smaller. In all tests of static and dynamic properties of rock and rock joints, dynamic values (Young modulus, shear modulus or joint stiffness) are invariably higher than static ones. Cai (2001) tested artificial cement mortar joints with 
compression stress from 2 to $20 \mathrm{MPa}$ and a central wave frequency of $20 \mathrm{kHz}$ under three growing deformation velocities: quasi-static, dynamic deformation on a dynamic press and ultrasonic waves. He found that dynamic stiffness on wave passage tests is typically $2-3$ times the value of dynamic deformation tests, which is itself 1.2-2 times the static stiffness. Cai seismic stiffness values are in the $20-1,200 \mathrm{GPa} / \mathrm{m}$ range depending on the compression and the density of contacts in the joint. The values found in the current study are in the same order of magnitude, increasing from 100 to $1,200 \mathrm{GPa} / \mathrm{m}$ for compression stresses of 1-120 MPa. On the other hand, values determined by Pyrak-Nolte et al. (1990) for transmission of waves across natural fractures on quartz monzonite compressed up to $85 \mathrm{MPa}$ yielded much larger seismic stiffness values, from 2,000 to $150,000 \mathrm{GPa} / \mathrm{m}$. However, the wave central frequency is higher, from 500 to $1,000 \mathrm{kHz}$. The static stiffness for the same samples is in the same order of magnitude in this study, but always lower ranging from 2,000 to $30,000 \mathrm{GPa} / \mathrm{m}$.

Even though no viscous parcel is present in the discrete element model as it was employed and no strain-rate effects are included in the contact models of the joint and rock material, dynamic elastic parameters are higher than static parameters. The reasons for this difference, also found in laboratory and field tests, are identical for fractures and for intact rock. First, due to inertia effects when rates of deformation are high, the fractures and asperities do not have enough time to deform. Second, the stress level induced by the dynamic wave is much smaller than the static stress. In the present study stress waves amplitude is approximately $0.1 \mathrm{MPa}$, which is one to three orders of magnitude smaller than the static stress installed in the joint. The wave with very small amplitude excites the fracture around the current location in the stress-displacement curve without perturbing the fracture state.

\section{Conclusions}

This paper describes an innovative method for modelling rock fracture dynamic behaviour using BPM, focusing on the propagation of compressive waves across fractures.

A simple method to generate a fracture in the BPM model is proposed and a contact constitutive with stiffness hardening, compiled in $\mathrm{C}^{++}$and linked to the source code of $\mathrm{PFC}^{2 \mathrm{D}}$, is employed in wall-to-wall interaction. The goal of this first part of the study is to reproduce the basic characteristics of a rock joint under normal load including the increase in the number of contact points and stiffness with stress. The limitations of this part of the study are also the ground for future developments. First, besides joint nonlinearity, also rock non-linear behaviour and failure can be integrated. Second, real joint profiles are extremely varied with peaks and valleys of different wavelengths and heights. The method used for the joint geometry generation does not reflect that, and the new contact model still has to be tested in other joint geometries. Third, though having a physical meaning, the contact model parameters do not correspond to rock joint walls measurable characteristics like hardness or resistance of the wall material. Finally, 3D modelling will allow for a better representation of the joints rich spatial variation. This is within reach if parallelized codes that run on multi-core or multi-processor machines are used.

On the second part of the work, the conditions for propagation of stress waves on rock bonded particle models are tested. The performance of dense non-uniform assemblies of particles is put side by side with known analytical results of organized packings and they are found to be demanding in terms of particles per wavelength but not enough to hinder the continuation of the work. A number of modelling details such as the transition from static to dynamic calculation modes, static and dynamic load conditions and absorbing boundary conditions are implemented. This shows that particle methods are no more a promising tool in rock mechanics but already a certainty, and a very adequate one for dynamic analyses.

The final part of the study is the simulation of stress wave interaction with rock fractures. Compressive waves with wide frequency content are sent into compressed fractures and the resulting reflected and transmitted waves are captured and compared with the theoretical solutions. The first success to mention is that the physical phenomenon is consistently reproduced. Both transmitted and reflected waves are captured with good quality, energy conservation is verified, and dynamic fracture stiffness values calculated from the DDT for both waves are in agreement. Moreover, when dynamic and static joint stiffnesses are compared, the former shows to be higher, as in laboratory studies.

The joint geometric representation is still limited by model size constraints. The major immediate task ahead is the implementation of more realistic joint geometries and contact models that simulate with more accuracy the mechanics of asperity deformation. In addition, joint filling or weathering is sometimes encountered in nature and they alter the joint behaviour. Strategies to simulate their effect can be devised.

All tests should be also carried out with shear waves in order to calculate the dynamic shear joint stiffness as well as the rock dynamic shear and bulk moduli. Other waveforms can also be used and the consistency of the results checked. The study of waves oblique to the fracture will demand larger models and more sophisticated methods for wave extraction and separation but will also allow for the complete verification of the DDT. 
Mixed static compression and shear loading are the general condition of joints in nature, and higher degrees of joint degradation may result, which affects their static and dynamic properties. The BPM should be able to simulate this.

Finally, the expansion to three dimensions will also allow for greater realism not only on the joint surface representation, but also of the waves, which can then have different polarizations in relation to the joint.

Acknowledgments The authors wish to thank the Laboratory of Rock Mechanics of the Ecole Polytechnique Fédérale de Lausanne for hosting the first author during part of the study. This work was funded by the Portuguese National Laboratory for Civil Engineering and the Foundation for Science and Technology (FCT) through project grant POCTI/ECM/57495/2004 and PhD grant SFRH/BD/36212/2007.

\section{References}

Abe S, Place D et al (2004) A parallel implementation of the lattice solid model for the simulation of rock mechanics and earthquake dynamics. Pure Appl Geophys 161(11):2265-2277

Achenbach JD (1973) Wave propagation in solids. North Holland, Amsterdam

Bandis SC, Lumdsen AC et al (1983) Fundamentals of rock joint deformation. International Journal of Rock Mechanics and Mining Sciences \& Geomechanics Abstracts 20(6):249-268

Belytschko T (1983) An overview of semidiscretization and time integration procedures. In: Belytschko T, Hughes TJR (eds) Computational methods for transient analysis. North Holland, Amsterdam, pp 1-65

Cai JG (2001) Effects of parallel fractures on wave attenuation in rock. School of Civil and Structural Engineering, $\mathrm{PhD}$ thesis. Nanyang Technological University, Singapore, p 306

Cundall PA (2000) Numerical experiments on rough joints in shear using a bonded particle model. Aspects of Tectonic Faulting (Festschrift in Honnour of Georg Mandl). Springer, Berlin

Cundall PA, Strack ODL (1979) A discrete numerical model for granular assemblies. Geotechnique 29(1):47-65

Donzé FV, Bernasconi P (2004) Simulation of blast patters in shaft sinking using Discrete Element Method. Electr J Geotech Eng Geol 9(B):22

Donzé FV, Bouchez J et al (1997) Modeling fractures in rock blasting. International Journal of Rock Mechanics and Mining Sciences 34(8):1153-1163

Duffy J (1959) A differential stress-strain relation for the hexagonal close-packed array of elastic spheres. Annual Meeting of the American Society of Mechanical Engineering. ASME, New York

Fan SC, Jiao YY et al (2004) On modelling of incident boundary for wave propagation in jointed rock masses using discrete element method. Comput Geotech 31(1):57-66

Gutierrez M, Barton N (1994) Numerical modelling of the hydromechanical behavior of single fractures. In: 1st North-American Rock Mechanics Symposium, Texas

Hazzard JF, Young RP (2004) Numerical investigation of induced cracking and seismic velocity changes in brittle rock. Geophys Res Lett 31:4

Hentz S, Donzé FV et al (2004) Discrete element modelling of concrete submitted to dynamic loading at high strain rates. Comput Struct 82(29-30):2509-2524
Hoover WG, Ashurst WT et al (1974) Two-dimensional computer studies of crystal stability and fluid viscosity. J Chem Phys 60(10):4043-4047

Kabeya KK (1999) Investigation into the peak shear behaviour of rock joints using physical and numerical modeling. In: Proceedings of the 37th US Rock Mechanics Symposium-Rock Mechanics for Industry, Vail, Colorado, Balkema

Karami A, Stead D (2008) Asperity degradation and damage in the direct shear test: a hybrid FEM/DEM approach. Rock Mechanics and Rock Engineering 41(2):229-266

Kim MK, Kim SE et al (1997) A study on the behavior of rock mass subjected to blasting using modified distinct element method. Int J Rock Mech Mining Sci 34(3-4):156.e1-156.e14

Kim S, Jeong W et al (2006) Numerical simulation of blasting at tunnel contour hole in joint rock mass. Tunn Undergr Space Technol 21(3-4):306-307

Kusumi H, Matsuoka T et al (2005) Simulation analysis of shear behavior of rock joint by distinct element method. Eurock 2005-Impact of Human Activity on Geological Environment, Brno, Taylor and Francis

Lemos JV (1987) A distinct element method for dynamic analysis of jointed rock with application to dam foundation and fault motion. Department of Civil and Mineral Engineering, University of Minnesota, PhD thesis, p 295

Lysmer J, Kuhlemeyer AM (1969) Finite dynamic model for infinite media. Journal of the Engineering Mechanics Division of the American Society of Civil Engineers 95(4):859-869

Matsuoka T, Kusumi H et al (2003) Simulation of Hopkinson effect by discrete element method. ISRM 2003-technology roadmap for rock mechanics, South Africa

Min K-B, Jing L et al (2005) Effect of stress on mechanical and hydraulic rock mass properties-application of DFN-DEM approach on the data from site investigation at Forsmark, Sweden. In: Eurock 2005-Impact of human activity on the geological environment. Balkema, Brno

Mindlin RD (1954) Mechanics of granular media. In: Proceedings of the Second US National Congress of Applied Mechanics

Nolte DD, Pyrak-Nolte LJ, Beachy J, Ziegler C (2000) Transition from the displacement discontinuity limit to the resonant scattering regime for fracture interface waves. International Journal of Rock Mechanics and Mining Sciences 37:219-230

Park E-S, Martin CD et al (2004) Numerical simulation of the mechanical behavior of discontinuous rock masses. In: Numerical methods in micromechanics via particle methods. Balkema, Kyoto

Potyondy DO, Cundall PA et al (1996) Modelling rock using bonded assemblies of circular particles. Rock Mechanics US

Potyondy DO, Cundall PA (2004) A bonded-particle model for rock. International Journal of Rock Mechanics and Mining Sciences 41(8):1329-1364

Pyrak-Nolte L, Nolte DD (1992) Frequency dependence of fracture stifness. Geophys Res Lett 19(3):4

Pyrak-Nolte LJ, Myer LR et al (1990) Transmission of seismic waves across single natural fractures. J Geophys Res 95(B6):86178638

Schlangen E, van Mier JGM (1994). Experimental and numerical study of crack propagation in sandstone. In: Rock mechanics in petroleum engineering-Eurock 94. Balkema, Delft

Schoenberg M (1980) Elastic wave behavior across linear slip interfaces. Journal Acoustic Society of America 68(5):15161521

Tai Q, Sadd MH (1997) A discrete element study of the relationship of fabric to wave propagational behaviours in granular materials. International Journal for Numerical and Analytical Methods in Geomechanics 21(5):295-311 
Toomey A (2001) Particle-based numerical modelling of seismic wave propagation in fractured rock. PhD thesis, Department of Geology, National University of Ireland, Dublin, p 265

Toomey A, Bean C (2000) Numerical simulation of seismic waves using a discrete particle scheme. Geophysical Journal International 141(3):595-604
Zhao J, Cai JG (2001) Transmission of Elastic P-waves across Single Fractures with a Nonlinear Normal Deformational Behavior. Rock Mechanics and Rock Engineering 34(1):3-22 